\title{
The Fragile X Mental Retardation Protein protects the lung from xenobiotic stress by facilitating the Integrated Stress Response
}

\author{
Deblina Sain Basu' ${ }^{1,2}$, Rital Bhavsar ${ }^{1}$, Imtiyaz Gulami ${ }^{1,2}$, Sai Manoz \\ Lingamallu $^{1,6}$, Ravi Muddashetty ${ }^{1}$, Chandrakanth Veeranna ${ }^{3}$, Sumantra Chattarji1 ${ }^{1,4,5}$, \\ Rajesh Thimmulappa ${ }^{3}$, Aditi Bhattacharya ${ }^{1,4}$ and Arjun Guha ${ }^{1 *}$
}

1. Institute for Stem Cell Science and Regenerative Medicine (inStem), GKVK Campus, Bangalore -560065

2. Trans Disciplinary University, Yelahanka, Bangalore

3. JSS Medical College, JSS Academy of Higher Education \& Research, Mysore570015

4. Centre for Brain Development and Repair (CBDR), inStem, GKVK Campus, Bangalore -560065

5. National Centre for Biological Sciences, GKVK Campus, Bangalore- 560065

6. Manipal Academy of Higher Education, Madhav Nagar, Manipal-576104

*Author to whom correspondence may be addressed: arjung@instem.res.in

Keywords: Stress Response, Lung, Integrated Stress Response, FMR1, FMRP 


\section{ABSTRACT}

2 Stress response pathways protect the lung from the damaging effects of environmental

3 toxicants. Here we investigate the role of the Fragile $X$ Mental Retardation Protein

4 (FMRP), a multifunctional protein implicated in stress responses, in the lung. We report

5 that FMRP is expressed in murine and human lungs, in the airways and more broadly.

6 Analysis of airway stress responses in mice and in a murine cell line ex vivo, using the

7 well-established Naphthalene (Nap) injury model, reveals that FMRP-deficient cells

8 exhibit increased expression of markers of oxidative and genotoxic stress and increased

9 cell death. We find that FMRP-deficient cells fail to actuate the Integrated Stress

10 Response Pathway (ISR) and upregulate the transcription factor ATF4. Knockdown of

11 ATF4 expression phenocopies the loss of FMRP. We extend our analysis of the role of

12 FMRP to human bronchial BEAS-2B cells, using a 9, 10-Phenanthrenequinone air

13 pollutant model, to find that FMRP-deficient BEAS-2B also fail to actuate the ISR and

14 exhibit greater susceptibility. Taken together, our data suggest that FMRP has a

15 conserved role in protecting the airways by facilitating the ISR. 


\section{INTRODUCTION}

17 The epithelial lining of the respiratory tract is continually challenged by a diverse array of environmental toxicants including gases, particulates, and other biological agents. Exposure to these agents leads to increased oxidative, genotoxic and endoplasmic reticulum stress. Such stresses, when unmitigated, lead to cellular damage, inflammation and in the long term to decreased lung capacity and functionality. The aim of this study was to probe the mechanisms by which lungs cope with environmental stresses.

The capacity of the lung to manage xenobiotic stress is dependent on stress response proteins that are induced upon insult. In this regard, the Integrated Stress Response (ISR) pathway is an evolutionarily conserved pathway that is integral to how the lung copes with environmental challenges (Pakos-Zebrucka K et al., 2016; van 't Wout EF et al., 2014; Konsavage WM et al., 2012). The ISR is triggered by the activation of one or more of the four stress-responsive kinases GCN2, PKR, PERK and HRI. The activation of these kinases, in turn, sets in motion two separate but interdependent processes that enable cells to mount a restorative response (Wong HR \& Wispe JR, 1997). First, these kinases phosphorylate the Eukaryotic Initiation Factor $2 \alpha$ (elF2 $\alpha)$ and shut off ongoing programs of protein synthesis. The inhibition of translation leads to the sequestration of translationally active mRNAs into stress granules (SGs). Second, activation of the kinases also induces specialized modes of protein translation leading to the expression of stress response proteins. More specifically, these specialized translation regimes upregulate expression of Activating Transcription Factor 4 (ATF4) (Pakos-Zebrucka K et al., 2016; van 't Wout EF et al., 2014) and, in turn, ATF4 targets such as ATF3. ATF4 also synergizes with other transcription factors activated in response to stress like Nrf2, to induce the expression of other stress response genes (He CH et al., 2001; Sarcinelli C et al., 2020).

The Fragile $X$ Mental Retardation Protein (FMRP) is a multifunctional protein that is expressed in the brain and more widely, in humans and other mammals alike. Deficiencies in FMRP lead to Fragile X Mental Retardation Syndrome (FXS), a disease characterized by mild-to-moderate intellectual disability (Zhou Z et al., 2014). FMRP 
has been shown to regulate synaptic plasticity by multiple mechanisms (Santoro MR et al., 2012). Aside from this well-established role, several studies indicate that FMRP also has a role in facilitating stress responses. At a cellular level, FMRP has been shown to play an essential role in SG biogenesis in response to arsenite and heat shock-induced stress (Didiot MC et al., 2009; Linder B et al., 2008). A recent study on fibroblasts in FMR1 $\mathrm{KO}$ mice showed that these cells are unable to mount a DNA Damage Response (DDR) when exposed to agents like Aphidicolin, 5-Hydroxyurea (5-HU) and UV but are able to do so in response to other types of DNA damaging agents (Alpatov $R$ et al., 2014). The central finding of this study is that FMRP has a chromatin-dependent role in resolving stalled replication forks and single strand breaks in DNA (Alpatov R et al., 2014). The environmental toxicants that the lung is exposed to typically induce a wide spectrum of genotoxic perturbations. Whether the chromatin-dependent role of FMRP is essential in this milieu is not clear.

59 Our interest in candidate proteins that regulate the pulmonary stress response led us to explore the role of FMRP in the lung. Immunostaining of murine and human lungs revealed that the protein is expressed in the airway epithelium and more broadly. To

62 probe the role of FMRP in stress responses in the airways, we subjected Fmr1 KO mice to Naphthalene injury, a well-established model for oxidative and genotoxic stress. We found that the airways of Fmr1 KO mice exhibited higher expression of markers of

65 oxidative and genotoxic stress, and greater cell death, than wild type. These findings led us to investigate the role of FMRP in airway stress responses in mice, the involvement of

67 the protein in the ISR, and its role in the human lung. 
FMRP is expressed in the airways and more broadly in the murine lung and protects airway Club cells from Naphthalene induced stress

To characterize the role of FMRP in the pulmonary stress response, we examined the expression of the protein in adult lungs from wild-type (WT) and Fmr1 KO animals. Lung sections from WT mice were stained with anti-FMRP antisera and examined under a confocal microscope (5 um, n>3 mice). FMRP expression was detected throughout the lung (Fig. 1A, 1C). We detected widespread protein expression in airway epithelium, both in secretory Club cells (CCs, marked by expression of Scgb1a1, Fig. 1A, 1C) and in ciliated cells (marked by expression of Acetylated Tubulin, AcTub). Outside of the airways, we noted intermittent expression in the alveolar parenchyma (Fig. 1A). Lung sections of Fmr1 KO mice stained with the same anti-FMRP antisera did not show any specific staining (airways shown in Fig. 1B, 1D, $n>3$ mice). Together, these experiments showed that FMRP is expressed in the murine lung, in the airways and more broadly. We also examined H\&E stained lung sections from WT and Fmr1 KO mice to find no obvious abnormalities in Fmr1 KO lungs (Fig. S1 A-B).

To investigate the role of FMRP in the pulmonary stress response, we focused our attention on FMRP-expressing airway CCs and exposed WT and Fmr1 KO mice to a chemical that targets CCs. Airway CCs are highly sensitive to the polycyclic hydrocarbon Naphthalene (Nap) (Stripp BR et al., 1995; Van Winkle LS et al., 1995). Nap administration leads to the loss of the vast majority of CCs from the airway epithelium within 24-48 $\mathrm{h}$ and is a well-established model for lung injury (Guha A et al., 2014; Guha A et al., 2017). The susceptibility of airway CCs to Nap is due to the expression, in CCs, of the cytochrome P450 enzyme Cyp2f2 (Buckpitt A et al., 2002). Cyp2f2 converts Nap to Naphthalene oxide that causes DNA damage. Naphthalene oxide is also converted to

93 Naphthoquinones that cause oxidative stress (Buckpitt A et al., 2002). The Cyp2f2 isoform that converts Nap to cytotoxic derivatives is not expressed in humans and consequently Nap does not affect humans in the same way. 
We exposed WT and Fmr1 KO animals to Nap and harvested lungs for analysis at different timepoints post injury (regimen shown schematically in Fig. 1E). To assess the extent of injury, we quantified frequencies of CCs across timepoints and examined expression of markers of oxidative and genotoxic stress. We found that the frequencies of CCs in WT were significantly higher than in Fmr1 KO at $24 \mathrm{~h}$ and $48 \mathrm{~h}$ respectively (Fig. 1F, n=3 mice per genotype per timepoint). In other words, cell loss was greater in Fmr1 KOs. Next, we stained sections from mouse lung both prior to and post Nap injury with two antisera: anti-4-Hydroxynonenal (4HNE, a product of lipid peroxidation and a marker of oxidative stress) and anti- $\mathrm{y}-\mathrm{H} 2 \mathrm{AX}$ (a phosphorylated histone variant that is a marker of double stranded DNA breaks and genotoxic stress). We did not detect expression of either stress marker in the lung in uninjured WT and Fmr1 KO mice (Fig. S1C, S1D, also Fig. 1G i- 1G ii, $1 \mathrm{H} \mathrm{i} \mathrm{-} \mathrm{1H} \mathrm{ii)} \mathrm{and} \mathrm{the} \mathrm{expression} \mathrm{of} \mathrm{both} \mathrm{markers} \mathrm{was}$ dramatically increased in Nap-injured lungs. Pertinently, we noted that the frequencies of CCs were higher and the levels of $4 \mathrm{HNE}$ and $\mathrm{y}-\mathrm{H} 2 \mathrm{AX}$ expression were lower in WT than in Fmr1 KOs, at all timepoints examined (Fig. 1G iii -1G vi,1H iii -1H vi, Fig. S1, n=3 mice per genotype per timepoint). Based on these data we concluded that CCs in Fmr1 KO animals are more susceptible to Nap-induced stress.

The Club cell-like C22 cell line deficient in FMRP is also more susceptible to Nap induced stress

115 To further probe the role of FMRP in stress responses in CCs, we turned to the murine Club cell-like cell line, C22. C22 cells were isolated from $\mathrm{H}$-2Kb-tsA58 mice expressing a

117 temperature sensitive isoform of the SV40 Large T antigen under the $\mathrm{H}-2 \mathrm{~Kb}$ promoter 118 (Demello DE et al., 2002). To characterize these cells, we stained C22 cells with markers 119 of CCs and other airway and alveolar lineages. Consistent with results from previous 120 reports, these cells expressed the CC marker Scgb1a1 and did not express markers of 121 other lineages (Fig. 2A, data not shown). To determine whether C22 could be utilized as 122 a model for Nap injury, and to probe the role of FMRP therein, cells were stained with 123 antisera against Cyp2f2 and FMRP. We found that C22 cells expressed modest levels 124 of Cyp2f2 (Fig. 2B) and also expressed FMRP (Fig. 2C, n>6 experiments). 
125

126

127

128

129

130

131

132

133

134

135

136

137

138

139

140

141

142

143

144

145

146

147

148

149

150

151

152

153

154

Next, we optimized methods for challenging C22 cells with Nap and for the knockdown of FMRP expression in these cells via RNA interference. Careful titration of Nap dosage and time of exposure (see methods) showed that a $1 \mathrm{~h}$ pulse of Nap was sufficient to induce expression of oxidative and genotoxic stress markers in C22 cells and marginally increase cell death $24 \mathrm{~h}$ post exposure. In an independent set of experiments, we established that treatment with 3 different Fmr1 siRNAs was sufficient to reduce FMRP levels expression by $80 \%$ or greater (see methods, compare FMRP expression in scrambled siRNA-treated cells, Sc, and Fmr1 siRNA-treated cells, Si in Fig. 2D, n>3 experiments).

To determine if FMRP regulates susceptibility to Nap in C22 cells, we incubated control (scrambled siRNA-treated cells, Sc) and FMRP-depleted (Fmr1 siRNA-treated cells, Si) cells with Nap for $1 \mathrm{~h}$ and the harvested cells at different timepoints for analysis (shown schematically in Fig. 2E, see methods). To assess levels of oxidative and genotoxic stress, we stained cells with anti-4HNE and anti- $\mathrm{H}-\mathrm{H} 2 \mathrm{AX}$ respectively (Fig. 2F-2H). To assess Nap cytotoxicity, cells were subject to a WST-1 assay $24 \mathrm{~h}$ post exposure. We found that levels of $4 \mathrm{HNE}$ and $\mathrm{Y}-\mathrm{H} 2 \mathrm{AX}$ (Fig.2G-2I) were elevated in Fmr1-depleted cells at all timepoints ( $n=3$ experiments each) and that Fmr1-depleted cells exhibited greater cell death in response to Nap (Fig. 2J). These data correlated well with the increased susceptibility of CCs to Nap in Fmr1 KO animals and demonstrated that FMRP has a cell intrinsic role in protecting cells from Nap.

FMRP is required for the induction of the Integrated Stress Response pathway that protects from Naphthalene induced stress

The increased susceptibility of FMRP-deficient CCs and C22 cells to Nap led us to investigate further the role of FMRP in the Nap induced stress response. As mentioned previously, FMRP has been shown to regulate the formation of SGs in response to stress. SG biogenesis is an integral aspect of the stress response. However, it is currently unclear whether a defect in SG biogenesis alone can render cells more susceptible to stressful stimuli (Adjibade P et al., 2017). Nevertheless, in an effort to characterize the contribution of FMRP in the stress response, we probed SG biogenesis in control and 
FMRP-depleted C22 cells post Nap. TIA1, Atx2 and G3BP are integral components of SGs in mammalian cells (Anderson P \& Kedersha N, 2006; Anderson P \& Kedersha N, 2008). To determine whether the genesis of SGs was inhibited in FMRP-deficient C22 cells $1 \mathrm{~h}$ post Nap, we stained Sc and Si cells with antisera against TIA1/Atx2 (anti-TIA1 immunostaining shown in Fig. 3A, anti-Atx2 immunostaining shown in Fig. S2A). Confocal analysis showed that Sc cells contain a few sporadic SGs and the numbers of SGs increased dramatically 3, 6, and $12 \mathrm{~h}$ post Nap and returned to baseline by $24 \mathrm{~h}$ (Fig. 3A, Fig. S2A, $\mathrm{n}=3$ experiments). Analysis of $\mathrm{Si}$ cells showed that these cells contain a few SGs in the untreated condition and that the numbers of SGs did not increase post Nap (Fig. 3A). Based on this analysis, we inferred that SG biogenesis is perturbed in FMRPdeficient cells post Nap. Co-staining cells with markers of SG and FMRP showed that FMRP has a punctate distribution both before and after Nap and that FMRP punctae were largely distinct from SGs. Based on these findings we concluded that FMRP regulates $S G$ biogenesis in response to Nap.

In addition to a role in SG biogenesis, FMRP also has a chromatin-dependent role in resolving certain types of genotoxic stress. More specifically, FMRP-deficient cells fail to recruit $\mathrm{y}-\mathrm{H} 2 \mathrm{AX}$ to stalled replication forks and single strand breaks in response to Aphidicolin, 5HU and UV exposure but are able to recruit $\mathrm{Y}-\mathrm{H} 2 \mathrm{AX}$ in response to gammaradiation (Alpatov $\mathrm{R}$ et al., 2014). While it is plausible that FMRP serves a similar role in Nap-treated cells, we noted that the nuclear accumulation of $\mathrm{y}-\mathrm{H} 2 \mathrm{AX}$ in FMR-deficient CCs and C22 cells post Nap was greater than in the respective controls. This suggested to us that the DNA Damage Response was at least partially active in FMR-deficient cells and, more importantly, that extent of DNA damage (as reported by nuclear $\mathrm{y}-\mathrm{H} 2 \mathrm{AX}$ accumulation) was greater in FMR-deficient cells than in controls (see Discussion).Together, the data led us to hypothesize FMRP has a more general role in facilitating stress responses post Nap and led to investigate its role in the Integrated Stress Response (ISR) pathway.

As previously mentioned, the ISR is induced when one of four stress-responsive kinases (GCN2, PERK, HRI, PKR) phosphorylate elF2 $\alpha$ at Serine 51. Phosphorylation of elF2 $\alpha$ arrests conventional translation, stimulates genesis of SGs and enables specialized 
185

186

187

188

189

190

191

192

193

194

195

196

197

198

199

200

201

202

203

204

205

206

207

208

209

210

211

212

213

214

translation of stress response proteins like ATF4 (Pakos-Zebrucka K et al., 2016; van 't Wout EF et al., 2014). To probe the status of the ISR in C22 cells post Nap, we examined the phosphorylation state of elF2 $\alpha$. We exposed C22 cells to Nap for $1 \mathrm{~h}$, harvested cells at various timepoints and quantified the levels of expression of both elF2 $\alpha$ and phosphorylated elF2 $\alpha$ (p-elF2 $\alpha$ ). Western blot-based ratiometric quantitation of total and p-elF2 $\alpha$ in Sc cells showed that p-elF2 $\alpha$ levels increased 3 and $6 \mathrm{~h}$ post injury and decreased to baseline levels thereafter (Fig. 3B, Fig. S2B - S2C, $n=5$ experiments). We inferred that the ISR is induced in C22 in response to Nap. We then exposed Si cells to Nap for $1 \mathrm{~h}$ and found that, contrary to controls, the levels of $p$-elF2 $\alpha$ did not increase post Nap (Fig. 3B, Fig. S2B - S2C, $n=5$ experiments). The analysis of $p$-elF2 $\alpha$ suggested the FMRP-depletion might inhibit the ISR.

Next, we examined the expression of ATF4 and its target, ATF3, in control and FMRPdeficient cells. Sc and Si cells were stained with an anti-ATF4 antibody prior to and post Nap. In Sc, the expression of ATF4 was undetectable in untreated cells, increased dramatically 3, 6, $12 \mathrm{~h}$ post Nap treatment and then approached baseline levels at $24 \mathrm{~h}$ (Fig. 3C, Fig. S2D, $n=3$ experiments). In Si cells, levels of ATF4 were negligible in untreated cells and showed no appreciable increase post Nap. Next, we assayed ATF3 levels by quantitative real-time PCR (qPCR). For this, RNA was isolated from Sc and Si cells at different timepoints and subjected it to qPCR analysis. In Sc, levels of ATF3 mRNA increased at 3, $6 \mathrm{~h}$ post Nap and returned to baseline thereafter (Fig. 3D, n=3 experiments). In Si, the levels ATF3 did not rise appreciably above baseline post Nap (Fig. 3D). These findings were also validated with anti-ATF3 immunostaining (data not shown). Based on these data we concluded that both ATF4 and ATF3 expression are perturbed in FMRP-deficient cells post Nap. Taken together, the findings showed that the ISR is perturbed in FMRP-deficient cells post Nap.

Next, we decided to investigate whether the upstream kinases that phosphorylate elF2 $\alpha$ and induce the ISR are activated (phosphorylated) in FMRP-deficient cells. We probed the expression of GCN2, PERK, HRI, PKR and their phosphorylated isoforms in Naptreated C22 cells using commercially available antibodies (see methods). Among all pairs of antisera tested, antisera for PKR and p-PKR provided reproducible results. Western 
215

216

217

218

219

220

221

222

223

224

225

226

227

228

229

230

231

232

233

234

235

236

237

238

239

240

241

blot-based ratiometric quantitation of p-PKR and total PKR in Sc and Si cells showed that the p-PKR levels increase in both Sc and SI 3 h post Nap (Fig. 3E, Fig. S2E - S2F, n=3 experiments). Importantly, we noted that levels of $p-P K R$ returned to baseline in Sc at 6 $\mathrm{h}$ and later timepoints, but remained significantly higher in Si at later timepoints (Fig. 3E). This suggested that at least one of the stress responsive kinases (PKR) is activated in FMRP-deficient cells post Nap but is unable to actuate downstream processes.

Perturbations in the ISR in FMRP-deficient C22 cells post Nap provided a plausible explanation for why these cells are more susceptible to Nap. To test this, we decided to probe how perturbing the ISR, by knocking down levels of Atf4, would impact susceptibility to Nap. Control (Scrambled siRNA) and Atf4 siRNA treated C22 cells were exposed to Nap as described earlier and cells were harvested at different timepoints for analysis. ATF4 immunostaining of control and Atf4 siRNA-treated cells showed that siRNA treatment eliminated ATF4 expression in cells post Nap exposure (Fig. 3F). We also found that ATF4-depleted cells exhibited increased expression of 4HNE (Fig. 3G, representative images shown in Fig. S2G) and $\mathrm{Y}-\mathrm{H} 2 \mathrm{AX}$ (Fig. 3H, representation images shown in Fig. $\mathrm{S} 2 \mathrm{H}$ ) at all timepoints examined ( $\mathrm{n}=3$ experiments each, quantitation of cell fluorescence based on $n>25$ cells per experiment) and also increased cell death $24 \mathrm{~h}$ post injury (Fig. 3I). We concluded that the increased levels of oxidative and genotoxic stress and increased cytotoxicity observed in FMRP-deficient cells could be due to a failure to induce the ISR and upregulate ATF4.

In light of the findings in C22 cells, we examined whether perturbations to the ISR are also observed in FMRP-deficient CCs in Nap-treated mice. We counterstained sections from control and Fmr1 $\mathrm{KO}$ lungs post Nap with antisera to both ATF4 and ATF3. Although ATF4 immunostaining was inconclusive, we noted that the levels of ATF3 were negligible in CCs in the control lung and upregulated post Nap (Fig. S2I - S2J, sections from n=3 mice). Pertinently, the levels of ATF3 in CCs in Fmr1 KO did not increase post Nap. These results are consistent with a role for FMRP in the induction of ISR in CCs post Nap. 
FMRP is expressed in the airways of the human lung and protects human bronchial BEAS-2B cells from 9, 10-Phenanthrenequinone induced stress

The findings in the murine lung led us to ask whether FMRP has a conserved role in the human lung. To investigate this possibility, we first examined the distribution of FMRP in the human lung. Paraffin sections stained with FMRP antisera showed that FMRP is expressed throughout the airways and more broadly (Fig. 4A, $n=2$ sections each from $\mathrm{n}=5$ independent lung biopsies). Triple labeling experiments with markers for ciliated cells and CCs showed that FMRP is expressed in both ciliated and non-ciliated cells, including CCs. Based on the distribution of FMRP we surmised that the protein could play a role in the airways in the human lung as well.

252

The BEAS-2B cell line is derived from normal human airways. These cells do not express markers of ciliated cells and, akin to CCs, have characteristics of non-ciliated cells. We stained BEAS-2B cells with FMRP antisera to find that these cells expressed FMRP (Fig. $4 B, n>6$ experiments). We then proceeded to develop an assay to probe the role of FMRP in stress responses in these cells.

Since the susceptibility of airway CCs to Nap is not recapitulated in the human lung or in BEAS-2B cells (data not shown), we utilized a different injury model to probe the role of FMRP in stress responses in human cells. 9, 10-Phenanthrenequinone (PQ) is an air pollutant that is present at high levels in diesel exhaust particles and is known to trigger oxidative and genotoxic stress (Lavrich KS et al., 2018). As part of our characterization of $P Q$, we first exposed control (scrambled siRNA-treated cells) and FMRP-depleted (Fmr1 siRNA-treated cells) C22 cells to a pulse of PQ for $1 \mathrm{~h}$ (see methods) and harvested cells at different timepoints for analysis (shown schematically in Fig. S3A). Consistent with our findings in the Nap model, we found that FMRP-depleted C22 cells exhibited increased expression of $4 \mathrm{HNE}$ (Fig. S3B) and $\mathrm{y}-\mathrm{H} 2 \mathrm{AX}$ (Fig. S3C) and increased cell death $24 \mathrm{~h}$ post exposure (Fig. S3D). We then examined ATF4 expression to find that although ATF4 levels increased in Sc cells at $3 \mathrm{~h}, 6 \mathrm{~h}$ post $\mathrm{PQ}$, no expression was detected in Si cells (Fig. S3E). These experiments showed that PQ treatment does lead to oxidative and genotoxic stress and that, FMRP-deficient C22 cells are more susceptible. 
272 Next, we exposed control (scrambled siRNA-treated cells) and FMRP-depleted (FMR1

273 siRNA-treated cells) BEAS-2B cells to a pulse of $P Q$ for $1 \mathrm{~h}$ and harvested them at 274 different timepoints for analysis (shown schematically in Fig. 4D). We determined

275 independently that the protocol for the knockdown of FMRP in BEAS-2B cells lead to a $27690 \%$ reduction in the levels of FMRP post treatment (Fig. 4C, $n>3$ experiments). We found 277 that FMRP-depleted cells exhibited increased expression of 4HNE (Fig. 4E-4F) and $Y^{-}$ 278 H2AX (Fig. 4G-4H) at all timepoints examined ( $n=3$ experiments each, quantitation of cell 279 fluorescence based on $n>25$ cells per experiment) and increased cell death $24 \mathrm{~h}$ post 280 281 injury (see methods, Fig. 4I). These experiments showed that FMRP-deficient BEAS2B cells are more susceptible to $P Q$.

FMRP is required for the induction of the Integrated Stress Response pathway that 283 protects from 9, 10-Phenanthrenequinone induced stress Next, we determined whether FMRP is required for the induction of the ISR in BEAS-2B cells. As described previously, we probed the activation status of PKR (ratiometric quantitation of $p-P K R$ and total PKR in both control cells (Scrambled siRNA-treated, Sc) and FMRP-deficient cells (Fmr1 SiRNA-treated, Si) Fig. 5A, Supplementary Fig. 4A-4B, $n=3$ ), the phosphorylation status of elF2 $\alpha$ (Fig. 5B, Fig. S4C - S4D, $n=5$ ), the formation of SGs (Fig. 5C, Fig. S4E $n=3$ ), the levels of ATF4 induction (Fig. 5D-5E, $n=3$ ) and the levels of ATF3 induction (Fig. 5F, $n=3$ ) at different times post PQ. These experiments showed that although p-PKR levels were increased in both Sc and Si post $P Q$, all of the downstream processes of the ISR were perturbed in Si. We concluded that FMRP is required for the ISR in BEAS-2B cells post $P Q$.

Next, we investigated whether the loss of ATF4 would recapitulate the loss of FMRP post PQ. Control (Scrambled siRNA) and ATF4 siRNA treated BEAS-2B cells were exposed to $P Q$ as previously described and cells were harvested at different timepoints for analysis. Consistent with expectations, ATF4 siRNA-treated cells showed no anti-ATF4 immunostaining post PQ exposure (Fig. 5G, $n>3$ experiments). We found that ATF4depleted BEAS-2B cells exhibited increased expression of 4HNE (Fig. 5H, representative images shown in Fig. S4F) and $\mathrm{Y}-\mathrm{H} 2 \mathrm{AX}$ (Fig. 5I, representative images shown in Fig. 
bioRxiv preprint doi: https://doi.org/10.1101/2021.03.16.435743; this version posted March 17, 2021. The copyright holder for this preprint (which was not certified by peer review) is the author/funder. All rights reserved. No reuse allowed without permission.

$301 \mathrm{~S} 4 \mathrm{G}$ ) and increased cell death $24 \mathrm{~h}$ post injury (see methods, Fig. 5J). These data 302 indicated that the loss of ATF4 largely phenocopies the loss of FMRP in PQ-treated 303 BEAS-2B cells. 
305 The aim of this study was to probe the role of FMRP in stress responses in the lung. We report that FMRP plays an essential role in protecting the airways in mice, and potentially in humans, from the deleterious effects of xenobiotic stress. Our studies provide strong evidence that FMRP protects the lung by facilitating the induction of the ISR (see model, Fig. 6). In the paragraphs that follow we will discuss the plausible mechanism/s by which FMRP may regulate the ISR, the possibility that FMRP regulates stress response pathways in addition to the ISR, and the clinical implications of the findings reported here.

A major finding of our study is that FMRP is required for the actuation of the ISR pathway. More specifically, we find that the stress responsive kinase PKR is activated in FMRPdeficient cells but that the phosphorylation of the PKR substrate, elF2 $\alpha$, is perturbed. The mechanism by which FMRP regulates this step is currently unknown. The analysis of FMRP-binding proteins in neuronal and other tissues has identified numerous interacting partners. Interestingly, among these interacting partners are the proteins Caprin1 and G3BP that have independently been implicated in the induction of the ISR pathway in response to stress (Taha MS et al., 2020; Wu Y et al., 2016). Pertinently, both Caprin1 and G3BP1 have been shown to be important for elF2 $\alpha$ phosphorylation (Reineke LC et al., 2015). Thus, it is plausible that FMRP acts in concert with Caprin1 and G3BP1 to

322 facilitate elF2 $\alpha$ phosphorylation. Although elF2 $\alpha$ phosphorylation is an early event in the ISR pathway and perturbations at this stage are likely to affect all downstream processes, our data do not allow us to rule out the possibility that FMRP has independent roles in downstream processes. FMRP could, for example, also have an independent role in 326 stress granule biogenesis (Didiot MC et al., 2009; Linder B et al., 2008). Our future experiments will probe these possibilities.

Studies that have examined the role of FMRP vis-à-vis stress responses suggest that FMRP could protect cells from stress in myriad ways. For example, it has been demonstrated that FMRP plays a chromatin-dependent role in inducing the DDR. This could be relevant in the context of the lung. Along the same lines, there is also evidence 
333 (Bechara EG et al., 2009). Levels of SOD1 are reduced in the brains of Fmr1 KO animals.

334 Since SOD1 has an important role in protecting cells from stress, FMRP could alter the 335 susceptibility of tissues to stressful stimuli by altering the baseline levels of SOD1. To 336 investigate this possibility, we probed levels of SOD1 in the brain and lung using both 337 Western Blot and immunohistochemical approaches (Fig. S5A). Although we did observe 338 that SOD1 levels in the brain were lower in Fmr1 KO than wild type, the levels of SOD1 339 in the lung were comparable. Moreover, we also analyzed SOD1 levels in the bronchial 340 cell lines (C22 and BEAS-2B) with or without FMRP to find that SOD1 levels were 341 comparable (Fig. S5D - S5I). Taken together, these data show FMRP is unlikely to 342 regulate SOD1 expression in the lung. Nevertheless, the role of 343 FMRP in the DDR, and in the regulation of SOD1 expression, show that FMRP can contribute toward protecting tissues from stress by ISR-independent mechanisms as well.

345 Although FMRP is broadly expressed in humans and mice alike 346 (https://www.genecards.org/cgi-bin/carddisp.pl?gene=FMR1), historically, FMRP has 347 almost exclusively been studied in a neural context due to its connection with intellectual 348 disability. An important finding of this study is that it demonstrates a role for FMRP in the 349 lung and points to a potential vulnerability in individuals with an FMR1 deficiency. 350 Clinically, the bulk of the case studies on FXS patients are derived from geographic 351 regions where the load of pulmonary environmental stressors is low. Our study suggests 352 that individuals with FXS living in areas of higher pollutant load may be more susceptible 353 to lung damage/disease and FMRP status in the lung may be a strong correlate of 354 resilience to pulmonary insults. 


\section{MATERIALS AND METHODS}

356

357

358

359

360

361

362

363

364

365

366

367

368

369

370

371

\section{2}

373

374

375

377

378

376 cell lines were tested for mycoplasma contamination and found to be negative. BEAS-

All animal work reported here has been approved by the Internal Animal Users Committee (IAUC) and the Institutional Animal Ethics Committee (IAEC) at inStem. Any procedure that could conceivably cause distress to the animals employed pre-procedural anesthesia with isofluorane gas (Baxter Healthcare Corp.), delivered by an anesthetic vaporizing machine. All animals were monitored for signs of distress and euthanized if in distress. The analysis of human biopsies was approved by Institutional Ethics Committee of JSS Medical College.

\section{Mouse strains}

Fmr1 knockout mice (Mus musculus) strain was maintained on a C57B6/J background at the Centre for Brain Development and Repair (CBDR), inStem. Genotyping of the animals was done using established protocols (Bakker CE et al., 1994).

\section{Human samples}

Human (Homo sapiens) lung tissue was obtained from five subjects at autopsy by a forensic pathologist from JSS Medical College, Mysore. The cause of death was not attributed to lung trauma. Deidentified samples were fixed in $4 \%$ paraformaldehyde at 4 ${ }^{\circ} \mathrm{C}$ overnight, embedded in paraffin, and processed for immunohistochemical analysis.

\section{Cell lines and culture conditions}

Human lung (BEAS-2B) non-ciliated airway epithelial origin cell line was obtained from Johns Hopkins University (kind gift from Prof. S. Biswal) (Singh A et al., 2009). The murine Club cell line (C22) was purchased from ECACC, UK (cat no.07021401, \#07D022). Both

377 2B were grown in DMEM: F12K (Gibco, USA, 21127030) (1:1) media, supplemented with $10 \%$ FBS (Gibco,USA, 10082147) and Penicillin-Streptomycin (Gibco, USA, 1540122) at 
$37^{\circ} \mathrm{C}, 5 \% \mathrm{CO} 2 . \mathrm{C} 22$ cell line was maintained in a proliferative state as per suppliers' instructions and experiments were performed $24 \mathrm{~h}$ post differentiation. Experiments were conducted within 3rd to 7th passages for BEAS-2B and within 3rd to 12th passages for C22.

\section{Models for xenobiotic stress}

For Naphthalene (Nap) injury in mice, wild type or Fmr1 knockout mice aged ( $\geq 8$ weeks of age) were injected intraperitoneally with Corn Oil (vehicle, Sigma, USA, C8267) or with Nap dissolved in corn oil (300 mg kg-1, (Sigma, USA, 147141 ) using established protocols (Guha A et al., 2014; Guha A et al., 2012). Animals were sacrificed 12 h, 24 h, 48 h after injection for analysis.

To establish an assay for Nap injury in C22 cells, we first determined that these cells expressed the cytochrome Cyp2f2 that converts Nap to stress-inducing derivatives. Having established this, we tested a range of concentrations of Nap (50 ug ml$)^{-1}$ to $500 \mathrm{ug}$ $\mathrm{ml}^{-1}$, in DMSO/DMEM). Nap was found to be stable in solution at concentrations upto 100 ug $\mathrm{mL}^{-1}$ and unstable at higher concentrations leading to cell death within $3 \mathrm{~h}$ post exposure. Nap exposure at 50-75 ug ml-1 (DMSO/DMEM, DMSO final concentration $0.7 \%)$ for short $(1 \mathrm{~h})$ and long duration $(24 \mathrm{~h}$ ) led to a progressive increase in expression of stress markers and mild cytotoxicity after a $24 \mathrm{~h}$ period. To probe the effects of FMRP/ATF4 deficiency on susceptibility to Nap, cells were exposed to Nap at $75 \mathrm{ug} \mathrm{ml}^{-1}$ (DMSO/DMEM, DMSO final concentration $0.7 \%$ ) for a period $1 \mathrm{~h}$. Cells were then washed in PBS and chased for varying periods of time in complete medium.

It has been reported previously that 9,10-Phenanthrenequinone (PQ) causes a sharp decrease in the viability of BEAS-2B cells when administered to cells for $24 \mathrm{~h}$ at concentrations greater than $1 \mathrm{uM}$ (Koike $\mathrm{E}$ et al., 2014). We reconfirmed these findings and determined the LD50 dose to be 1.5 uM ((Sigma, USA, 275034), dissolved in DMSO/DMEM, DMSO final concentration $0.00002 \%)$. To probe the effects of FMRP/ATF4 deficiency on susceptibility to $P Q$, cells were exposed to $P Q$ at $1.5 \mathrm{uM}$ (DMSO/DMEM, DMSO final concentration $0.00002 \%$ ) for a period $1 \mathrm{~h}$. Cells were then 
washed with PBS and fresh complete media and chased for varying period's time in complete medium.

\section{siRNA based knockdown of FMR1/ATF4 expression}

Several studies have demonstrated that multiple siRNA administered together or sequentially work more efficiently for silencing gene expression than a single siRNA (Wang $Z$ et al., 2016; Fähling $M$ et al., 2009; Zhang $P$ et al., 20015; Hatch EM et al., 2010). For our studies we used 3 distinct siRNAs for each targeted gene. siRNAs were administered to cells sequentially, $12 \mathrm{~h}$ apart, to silence the gene expression. siRNA transfections were done with Lipofectamine 2000 (Thermofisher Scientific, USA, 11668027). All xenobiotic stress assays in C22 cells were performed $36 \mathrm{~h}$ after treatment with the last siRNA. C22 cells were transferred from proliferative to differentiationinducing media $12 \mathrm{~h}$ after the last Si RNA treatment and utilized for xenobiotic stress assays $24 \mathrm{~h}$ thereafter. All xenobiotic stress assays in BEAS-2B cells were performed 12 $\mathrm{h}$ after treatment with the last siRNA. All siRNAs were obtained from Ambion: murine Fmr1 (Ambion, USA, 4390771), murine Atf4 (Ambion, USA, 16708), human FMR1 (Ambion, USA, 4392420) and human ATF4 (Ambion, USA, 16708), and Scrambled (Negative control, Ambion, USA, 4390843). The assay IDs for each of siRNAs are as follows: Mouse Fmr1 siRNA (Assay ID: 5315, 5317, s66177), Human FMR1 siRNA (Assay ID: 5315, 5316, 5317), Mouse Atf4 siRNA (Assay ID: 160775, 160776, 160777), Human ATF4 siRNA (Assay ID: 122168, 122287, 122372).

\section{Cell Cytotoxicity assay}

C22 and BEAS-2B cells were inoculated into 96-well plate and treated with Nap or PQ for $1 \mathrm{~h}$, as described above, and harvested for analysis $24 \mathrm{~h}$ later. Cell viability was assayed using WST-1 reagent (Sigma, USA, 5015944001)). Briefly, cells were incubated with WST-1 for $4 \mathrm{~h}$ and absorbance readings were taken and analyzed as per manufacturer's protocols. Cytotoxicity percentage $=100 \times[(O D(450 \mathrm{~nm}-650 \mathrm{~nm})$ of untreated cells-OD (450nm-650nm) of treated cells)/ OD (450nm-650nm) of untreated cells]. 


\section{Histology, Immunofluorescence and Imaging}

Lungs were inflated with 4\% (wt/vol) Paraformaldehyde (Alfa Aesar, USA, 30525-89-4) in PBS and fixed for 8 hours at $4^{\circ} \mathrm{C}$. Fixed lungs were subsequently embedded in paraffin, sectioned (5 um) and processed for immunohistochemical analysis post heat-mediated antigen retrieval at $\mathrm{pH} 6.0$ (Vector Labs, USA) except sections stained with anti-SOD1 antisera that were subject to antigen retrieval at pH 9.0 (Vector Labs, USA). For cellular immunostaining, cells were seeded on coated coverslips $(0.1 \%$ gelatin, Sigma, USA, G9391, as per manufacturer's protocol). Post-treatment, cells were fixed with 4\% PFA for 30 minutes and blocked with $2 \%$ FBS, $0.2 \%$ BSA and $0.1 \%$ Triton X 100 in 1 X PBS for an hour and stained. Primary antibodies were diluted using the same blocking solution. Immunohistochemical analysis utilized the following antisera: rabbit anti-FMRP (Abcam, UK, 17722, 1:500), rabbit anti-FMR1 (Sigma, USA, 1:200), goat anti-Scgb1a1 (Santa Cruz, USA, Sc365992, 1:500), mouse anti-acetylated tubulin (Sigma, USA, T7451, 1:1000), mouse anti-4HNE (Abcam, UK, ab48506, 1:500), rabbit anti- $\mathrm{Y}$-H2AX (Novus biological, USA, NB100-384, 1:1000), mouse anti-Cyp2f2 (Santa Cruz, USA, 1:200), mouse anti-ATF4 (Sigma, USA, WH0000468M1, 1:200), goat-Anti-Tia1 (Santa Cruz, USA, SC1751, 1:200), and Alexa 488/568/647-conjugated donkey anti-mouse/rabbit/goat secondary antibodies (Invitrogen, USA, 1:300). Stained sections were mounted in ProLong Diamond (Invitrogen, USA, P36962). All samples were imaged on a FV3000 4laser confocal microscope or on a Zeiss LSM-780 (Carl Zeiss AG, Germany) laserscanning confocal microscope.

\section{Quantitative fluorescence microscopy}

Frequencies of Club cells/mm airway, and total cellular fluorescence in Club cells, in lung sections, were determined from single tiled optical sections acquired on a confocal microscope using ImageJ software. For Club cell frequency analysis, cells attached to the basement membrane were counted per section per animal. Total cellular fluorescence intensity was calculated by subtracting a "background" value per section from the integrated density per cell (outlined using the software) (for FMRP, 4HNE, $\mathrm{y}-\mathrm{H} 2 \mathrm{AX}$ and ATF4). The "background" value was determined by sampling integrated density of regions 
463

464

465

466

467

468

469

470

471

472

473

474

475

476

477

478

479

480

481

482

483

484

485

486

487

488

489

490

on the section devoid of cells. Total cellular fluorescence of C22 and BEAS-2B cells was estimated from single optical sections on a confocal microscope using ImageJ software. In all experiments involving C22 and BEAS-2B cells, $\geq 25$ cells were analyzed per timepoint, per experiment, $\mathrm{n}=3$ experiments. The images of Scgb1a1 and Cyp2 2 expression in C22 cells, and of stress granule markers in BEAS-2B and C22 cells, are maximum intensity projection images of z-stacks acquired on confocal microscope.

\section{Western blot analysis}

Protein was extracted from cell lysates using RIPA buffer (Thermofisher Scientific, USA, 89900) containing Sigmafast EDTA free protease inhibitor cocktail (Sigma, USA, s8830) and Phosstop (Merk, USA, 4906845001). Total protein was run on a 12\% SDS PAGE, transferred onto a nitrocellulose membrane (Amersham, UK, 10600002), and the membrane was stained with reversible MemCode (Thermofisher Scientific, USA, 24580) for total protein estimation (imaged on ImageQuant600 (Amersham, UK) and quantified using ImageJ). The membrane was subsequently de-stained, blocked with 5\% BSA (Sigma, USA, A9418) for $1 \mathrm{~h}$ and probed using the following primary antisera: rabbit antiPhospho-elF2 $\alpha$ (Ser51) (Cell Signalling Technology, USA, 9721S, 1:1000), mouse antielF2 $\alpha$ (Cell Signalling Technology, USA, 2103S, 1:1000), rabbit anti-Phospho-PKR (Sigma, USA, SAB4504517,1:3000), mouse anti-PKR (Santa Cruz, USA, Sc-6282, 1:1000), rabbit anti-GCN2 (Cell Signalling Technology, USA, 3302s), mouse antiPhospho-GCN2 (Cell Signalling Technology, USA, 3301S), rabbit anti-PERK (Cell Signalling Technology, USA, 3192s, 1:1000), rabbit anti-Phospho-PERK (Cell Signalling Technology, USA, 1379s), mouse anti-HRI (Santa Cruz, USA, sc-365239). Primary antisera was detected using the following secondary antisera: HRP-conjugated anti-rabbit (abcam, UK, 6721, 1:3000) and HRP-conjugated anti-mouse secondary (Invitrogen, USA, \# 62-6520 1:5000) antibodies and ECL (BioRad, USA, 1620177) and analyzed (imaged on ImageQuant600 (Amersham, UK) and quantified using ImageJ). The levels of elF2a, phospho-elF2 $\alpha$, PKR and Phospho-PKR were normalized to the total protein content of the respective lanes. 


\section{Quantitative PCR (qPCR) analysis}

492 RNA from cell lysates was extracted using Trizol (Invitrogen, USA, 15596018, as per 493 manufacturer's protocol) and QPCR was performed using the primers listed in the 494 following table. The qPCR assays were constituted with the Maxima SYBR green/ROX 495 qPCR Mastermix (2X) (Thermo Scientific, USA, K0221) and analyzed on a BioRad CFX3 496 real-time PCR system (BioRad, USA).

\begin{tabular}{|l|l|}
\hline Gene & Sequence \\
\hline ATF3 Human Forward primer & GTACCCAGGCTTTAGCATTA \\
\hline ATF3 Human Reverse primer & TTAATAGACAGTAGCCAGCG \\
\hline Beta actin Human Forward primer & AAAACTGGAACGGTGAAGGT \\
\hline Beta actin Human Reverse primer & ACAACGCATCTCATATTTGGAA \\
\hline ATF3 Mouse Forward primer & GAGATGTCAGTCACCAAGTC \\
\hline ATF3 Mouse Reverse primer & TCCAGTTTCTCTGACTCTTTC \\
\hline Beta actin Mouse Forward primer & CTTCCAGCAGATGTGGATCAG \\
\hline Beta actin Mouse Reverse primer & AAAACGCAGCTCAGTAACAGT \\
\hline
\end{tabular}

\section{Statistical Analysis}

498 Statistical significance of datasets was assessed using unpaired two-tailed t-tests post 499 Shapiro-Wilk tests for normality. Data were also analysed using a two-way ANOVA to 500 compare changes in two groups with respect to time, genotype and interaction 501 parameters. ANOVA data and normality test results for each figure are presented (Table 502 S1 - S9) in a tabular format. 


\section{ACKNOWLEDGEMENTS}

504 We thank Joseph Jomon, National Centre for Cell Science (NCCS), Pune for sharing 505 antibodies; Aditya Deshpande, inStem for assistance in animal experiments; Sarfaraz 506 Nawaz and Sudhriti Ghosh Dastidar, inStem for their assistance with biochemical 507 analyses; Harlin Kaur, Binita Dam, Arnab Karmakar, Saraswati Chavda and Mamta 508 Yadav for technical assistance; and the Central Imaging and Flow Cytometry Facility 509 (CIFF) and Animal Care and Resource Center (ACRC) Facility at Bangalore Life Science 510 Cluster (BLiSC) for their constant support.

\section{COMPETING INTERESTS}

512 The authors declare no competing interests.

\section{FUNDING}

514 This work was funded by inStem core funds and the Ramalingaswami Reentry Fellowship 515 (AG, RB), and fellowships from Department of Biotechnology (SML), Indian Council of 516 Medical Research (IG) and University Grants Commission-Council of Scientific \& 517 Industrial Research (DSB). 


\section{REFERENCES}

Adjibade P, St-Sauveur VG, Bergeman J, Huot ME, Khandjian EW, Mazroui R. (2017). DDX3 regulates endoplasmic reticulum stress-induced ATF4 expression. Sci. rep. 7, 1-2. doi: $10.1038 / s 41598-017-14262-7$

Alpatov R, Lesch BJ, Nakamoto-Kinoshita M, Blanco A, Chen S, Stützer A, Armache KJ, Simon MD, Xu C, Ali M, Murn J. (2014). A chromatin-dependent role of the fragile X mental retardation protein FMRP in the DNA damage response. Cell. 157, 869-881. doi: 10.1016/j.cell.2014.03.040

Anderson P \& Kedersha N. (2006). RNA granules. J. Cell. Biol. 172, 803-808. doi: 10.1083/jcb.200512082

Anderson P \& Kedersha N. (2008). Stress granules: the Tao of RNA triage. Trends Biochem. Sci. 33, 141-150. doi: 10.1016/j.tibs.2007.12.003

Bakker CE, Verheij C, Willemsen R, van der Helm R, Oerlemans F, Vermey M, Bygrave A, Hoogeveen A, Oostra BA, Reyniers E, De Boule K. (1994). Fmr1 knockout mice: a model to study fragile X mental retardation. Cell. 78, 23-33. doi:10.1016/0092-8674(94)90569-X

Bechara EG, Didiot MC, Melko M, Davidovic L, Bensaid M, Martin P, Castets M, Pognonec P, Khandjian EW, Moine H, Bardoni B. (2009). A novel function for fragile X mental retardation protein in translational activation. PLoS. Biol. 7, e16. DOI: 10.1371/journal.pbio. 1000016

Buckpitt A, Boland B, Isbell M, Morin D, Shultz M, Baldwin R, Chan K, Karlsson A, Lin C, Taff A, West J. (2002). Naphthalene-induced respiratory tract toxicity: metabolic mechanisms of toxicity. Drug. Metab. Rev. 34, 791-820. doi:10.1081/DMR-120015694

\section{de Diego-Otero Y, Romero-Zerbo Y, el Bekay R, Decara J, Sanchez L, Rodriguez-de} Fonseca F, del Arco-Herrera I. (2009). Alpha-tocopherol protects against oxidative stress in the fragile $\mathrm{X}$ knockout mouse: an experimental therapeutic approach for the Fmr1 deficiency. Neuropsychopharmacology. 34, 1011-1026. doi:10.1038/npp.2008.152

Demello DE, Mahmoud S, Ryerse J, \& Hoffmann JW. (2002). Generation and characterization of a conditionally immortalized lung clara cell line from the H-2Kb-tsA58 transgenic mouse. In. Vitro. Cell. Dev. Biol. Anim. 38, 154-164. doi:10.1290/10712690(2002)038<0154:GACOAC > 2.0.CO;2

Didiot MC, Subramanian M, Flatter E, Mandel JL, Moine H. (2009). Cells lacking the fragile X mental retardation protein (FMRP) have normal RISC activity but exhibit altered stress granule assembly. Mol. bio. cell. 20, 428-37. doi:10.1091/mbc.e08-07-0737

El Bekay R, Romero-Zerbo Y, Decara J, Sanchez-Salido L, Del Arco-Herrera I, Rodríguez-de Fonseca F, De Diego-Otero Y. (2007). Enhanced markers of oxidative stress, altered antioxidants and NADPH-oxidase activation in brains from Fragile $\mathrm{X}$ mental 
retardation 1-deficient mice, a pathological model for Fragile X syndrome. Eur. J. Neurosci.. 26, 3169-80. doi:10.1111/j.1460-9568.2007.05939.x

Fähling M, Mrowka R, Steege A, Kirschner KM, Benko E, Förstera B, Persson PB, Thiele BJ, Meier JC, Scholz H. (2009). Translational regulation of the human achaete-scute homologue-1 by fragile X mental retardation protein. J. Bio. Chem. 284, 4255-66. doi: 10.1074/jbc.M807354200

Guha A, Vasconcelos M, Zhao R, Gower AC, Rajagopal J, Cardoso WV. (2014). Analysis of Notch signaling-dependent gene expression in developing airways reveals diversity of Clara cells. PLoS. One. 9, e88848. doi:10.1371/journal.pone.0088848

Guha A, Deshpande A, Jain A, Sebastiani P, Cardoso WV. (2017). Uroplakin 3a+ cells are a distinctive population of epithelial progenitors that contribute to airway maintenance and post-injury repair. Cell rep. 19, 246-54.doi:10.1016/j.celrep.2017.03.051.

\section{Guha A, Vasconcelos M, Cai Y, Yoneda M, Hinds A, Qian J, Li G, Dickel L, Johnson} JE, Kimura S, Guo J, McMahon J, McMahon AP, and Cardosoa WV . (2012). Neuroepithelial body microenvironment is a niche for a distinct subset of Clara-like precursors in the developing airways. PNAS. 109, 12592-12597.

doi:10.1073/pnas.1204710109

Hatch EM, Kulukian A, Holland AJ, Cleveland DW and Stearns T.J. (2010). Cep152 interacts with Plk4 and is required for centriole duplication. Cell Biol. 191, 721-729. doi: 10.1083/jcb.201006049

He CH, Gong P, Hu B, Stewart D, Choi ME, Choi AM, Alam J. (2001). Identification of activating transcription factor 4 (ATF4) as an Nrf2-interacting protein. Implication for heme oxygenase-1 gene regulation. J. Biol. Chem. 276, 20858-20865. doi: 10.1074/jbc.M101198200

Koike E, Yanagisawa R, Takano H. (2014). Toxicological effects of polycyclic aromatic hydrocarbons and their derivatives on respiratory cells. Atmos. Environ. 97, 529-36. doi:10.1016/j.atmosenv.2014.04.003

Konsavage WM, Zhang L, Wu Y, \& Shenberger JS. (2012). Hyperoxia-induced activation of the integrated stress response in the newborn rat lung. Am. J. Physiol. Lung. Cell. Mol. Physiol. 302, L27-35.; doi:10.1152/ajplung.00174.2011.

Lavrich KS, Corteselli EM, Wages PA, Bromberg PA, Simmons SO, Gibbs-Flournoy EA, Samet JM. (2018). Investigating mitochondrial dysfunction in human lung cells exposed to redox-active PM components. Toxicol. Appl. Pharmacol. 342, 99-107. doi: 10.1016/j.taap.2018.01.024

Li G,Scull C, Ozcan L, and Tabas I. J. (2010). NADPH oxidase links endoplasmic reticulum stress, oxidative stress, and PKR activation to induce apoptosis. Cell Biol. 191, 1113-1125. doi:10.1083/jcb.201006121 
Lima-Cabello E, Garcia-Guirado F, Calvo-Medina R, el Bekay R, Perez-Costillas L, Quintero-Navarro C, Sanchez-Salido L, de Diego-Otero Y. (2016). An Abnormal Nitric Oxide Metabolism Contributes to Brain Oxidative Stress in the Mouse Model for the Fragile X Syndrome, a Possible Role in Intellectual Disability. Oxid. Med. Cell. Longev. 8548910. doi: $10.1155 / 2016 / 8548910$ PKR-dependent CHOP induction limits hyperoxia-induced lung injury. Am. J. Physiol.-Lung Cell. Mol. 300, L422-L429. doi:10.1152/ajplung.00166.2010

603

Pakos-Zebrucka K, Koryga I, Mnich K, Ljujic M, Samali A, Gorman AM. (2016). The integrated stress response. EMBO rep. 17, 1374-95. doi: 10.15252/embr.201642195.

Reineke LC, Kedersha N, Langereis MA, van Kuppeveld FJ, Lloyd RE. (2015).Stress granules regulate double-stranded RNA-dependent protein kinase activation through a complex containing G3BP1 and Caprin1. M. Bio. 6, e02486-14. doi: 10.1128/mBio.02486-14

Romero-Zerbo Y, Decara J, El Bekay R, Sanchez-Salido L, Del Arco-Herrera I, De Fonseca FR, De Diego-Otero Y. (2008). Protective effects of melatonin against oxidative stress in Fmr1 knockout mice: a therapeutic research model for the fragile $\mathrm{X}$ syndrome. $J$. Pineal. Res. 46, 224-234. doi :10.1111/j.1600-079X.2008.00653.x

Santoro MR, Bray SM, Warren ST. (2012). Molecular mechanisms of fragile X syndrome: a twenty-year perspective. Annu. Rev. Pathol. 7, 219-45 doi:10.1146/annurev-pathol-011811132457

Sarcinelli C, Dragic H, Piecyk M, Barbet V, Duret C, Barthelaix A, Ferraro-Peyret C, Fauvre J, Renno T, Chaveroux C, Manié SN. (2020). ATF4-Dependent NRF2 transcriptional regulation promotes antioxidant protection during endoplasmic reticulum stress. Cancers. 12, 569. doi:10.3390/cancers 12030569

Singh A, Ling G, Suhasini AN, Zhang P, Yamamoto M, Navas-Acien A, Cosgrove G, Tuder RM, Kensler TW, Watson WH, Biswal S. (2009). Nrf2-dependent sulfiredoxin-1 expression protects against cigarette smoke-induced oxidative stress in lungs. Free. Radic. Biol. Med. 46, 376-86. doi: 10.1016/j.freeradbiomed.2008.10.026

Solomon S, Xu Y, Wang B, David M, Schubert P, Kennedy D, Schrader JW. (2007). Distinct structural features of Caprin-1 mediate its interaction with G3BP-1 and its induction of phosphorylation of eIF $2 \alpha$, entry to cytoplasmic stress granules, and selective interaction with a subset of mRNAs. J. Mol. Cell. Bio. 27, 2324-2342. doi:10.1128/MCB.02300-06.

Song G, Napoli E, Wong S, Hagerman R, Liu S, Tassone F, Giulivi C. (2016). Altered redox mitochondrial biology in the neurodegenerative disorder fragile X-tremor/ataxia 
syndrome: use of antioxidants in precision medicine. Mol. Med. 22, 548-559. doi: 10.2119/molmed.2016.00122

Stripp BR, Maxson K, Mera R, \& Singh G. (1995). Plasticity of airway cell proliferation and gene expression after acute naphthalene injury. Am. J. Physiol. 269, L791-799. doi: 10.1152/ajplung.1995.269.6.L791

Taha MS, Haghighi F, Stefanski A, Nakhaei-Rad S, Kazemein Jasemi NS, Al Kabbani MA, Görg B, Fujii M, Lang PA, Häussinger D, Piekorz RP. (2020). Novel FMRP interaction networks linked to cellular stress. FEBS J. doi: 10.1111/FEBS.15443.

van 't Wout EF, Hiemstra PS, \& Marciniak SJ. (2014). The integrated stress response in lung disease. Am. J. Respir. Cell. Mol. Bio.l 50, 1005-1009.s doi: 10.1165/rcmb.2014-0019TR

Van Winkle LS, Buckpitt AR, Nishio SJ, Isaac JM, \& Plopper CG. (1995). Cellular response in naphthalene-induced Clara cell injury and bronchiolar epithelial repair in mice. Am. J. Physiol. 269, L800-818. doi:10.1152/ajplung.1995.269.6.L800

Wang Z, Sun W, Cao J, Cui H, Ma Z. (2016). Repeated Aurora-A siRNA Transfection Results in Effective Apoptosis of A549 Cells Compared to Single Transfection. Clin. Lab. 62, 697-703. doi: 10.7754/clin.lab.2015.150836

Wong HR \& Wispe JR. (1997). The stress response and the lung. Am. J. Physiol. 273, L1-9. doi: 10.1152/ajplung.1997.273.1.L1

Wu Y, Zhu J, Huang X, Du Z. (2016). Crystal structure of a dimerization domain of human Caprin-1: insights into the assembly of an evolutionarily conserved ribonucleoprotein complex consisting of Caprin-1, FMRP and G3BP1. Acta Crystallographica Section D: J. Struct. Bio. 72, 718-27. doi:10.1107/S2059798316004903

Zhang P, Abdelmohsen K, Liu Y,Tominaga-Yamanaka K, Yoon JH, Ioannis G, Martindale JL, Zhang Y, Becker KG, Yang IH, Gorospe M \& Mattson MP. (2015). Novel RNA- and FMRP-binding protein TRF2-S regulates axonal mRNA transport and presynaptic plasticity. Nat. Commun. 6, 8888. doi: 10.1038/ncomms9888 influenza A virus. Nat. Commun. 5, 3259. doi: 10.1038/ncomms4259 
Figure-1
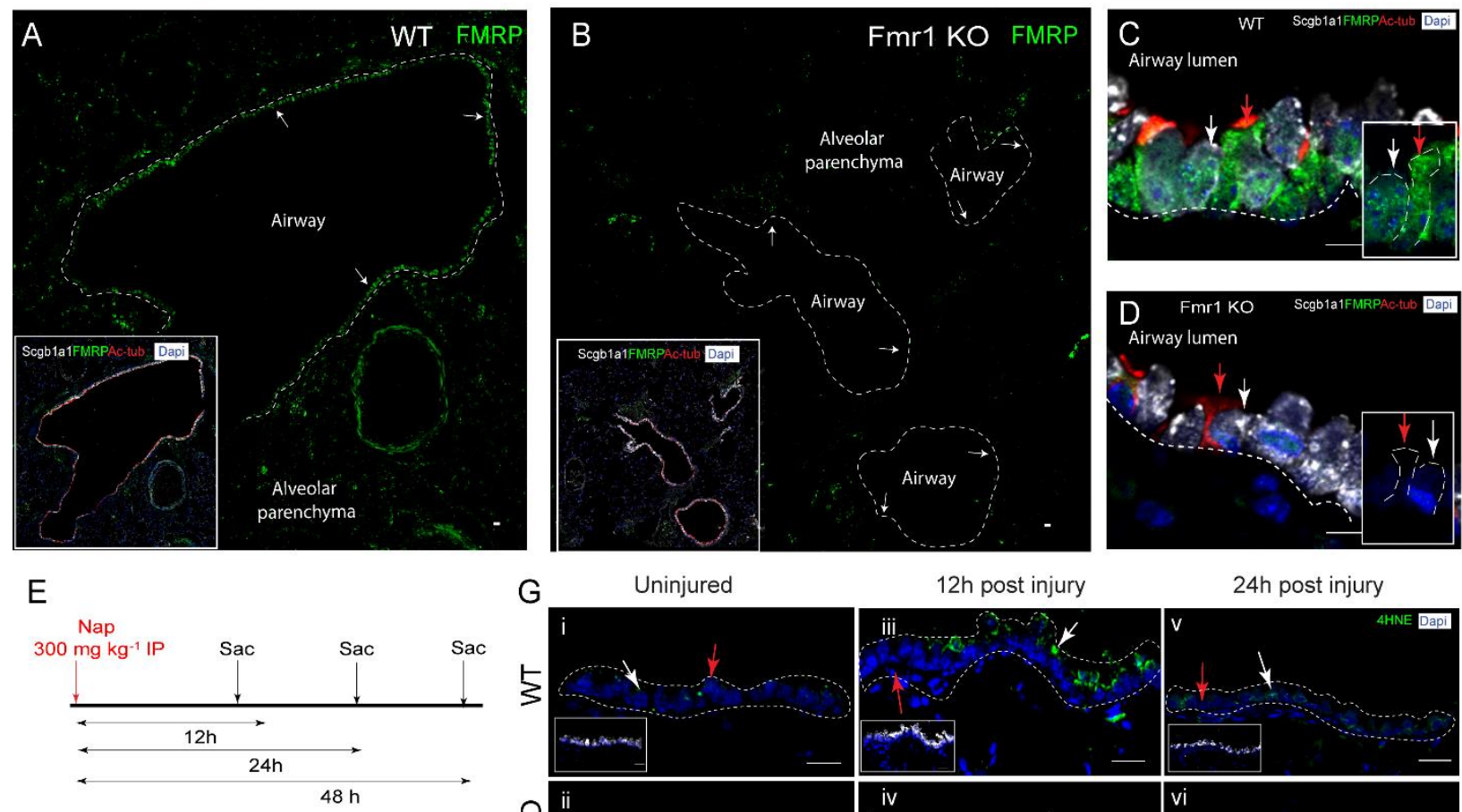

G

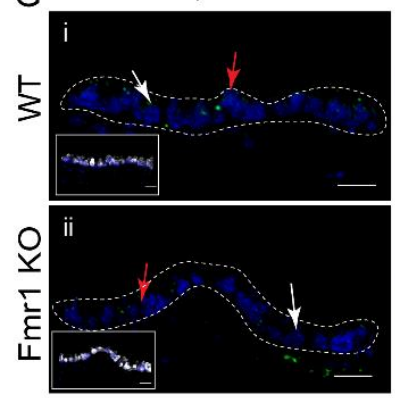

12h post injury
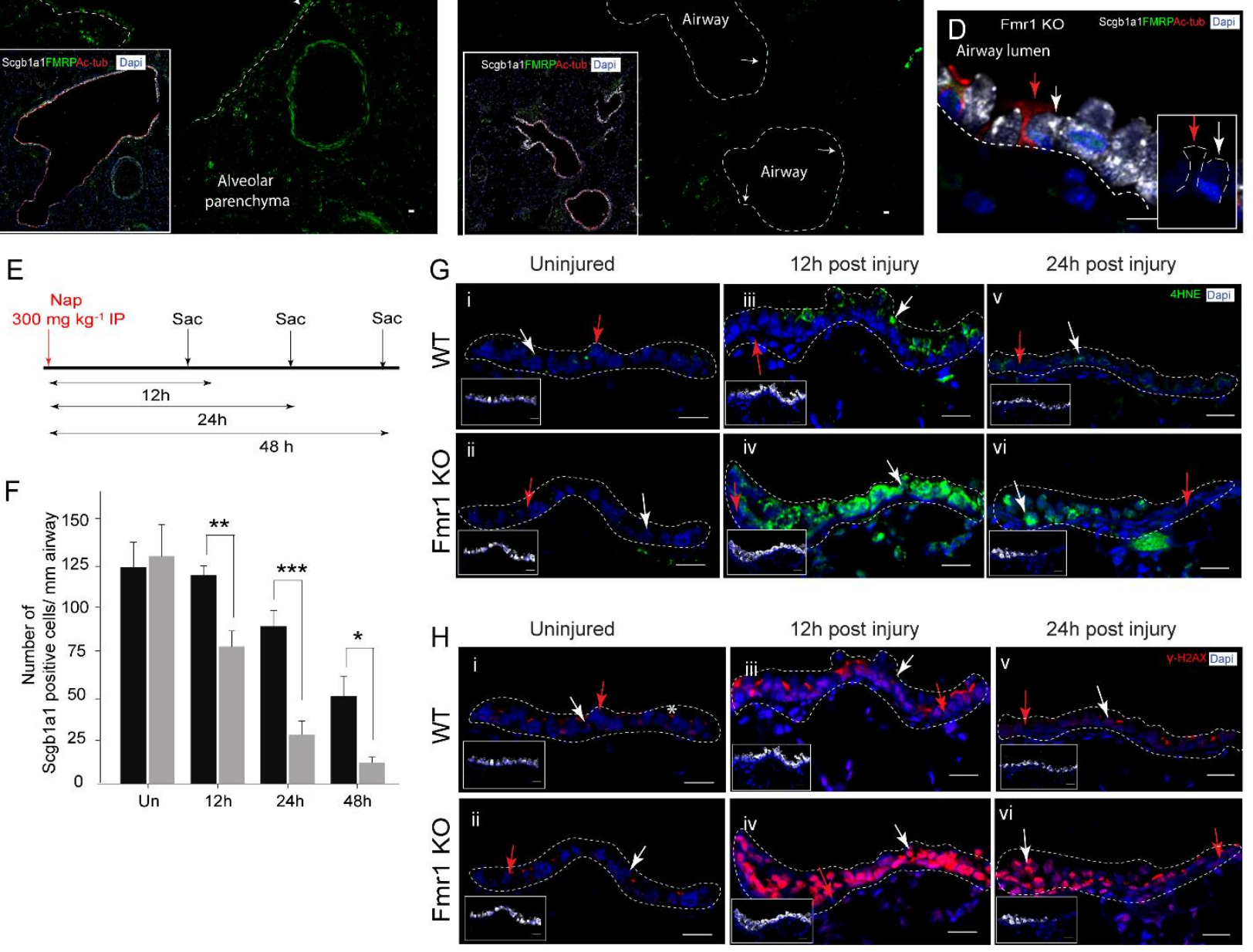

24h post injury

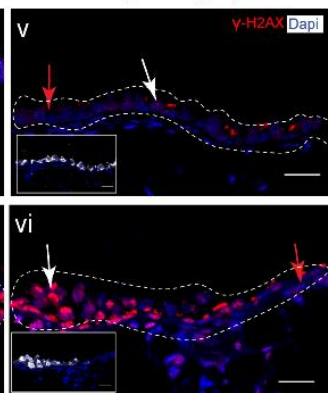


Figure-2
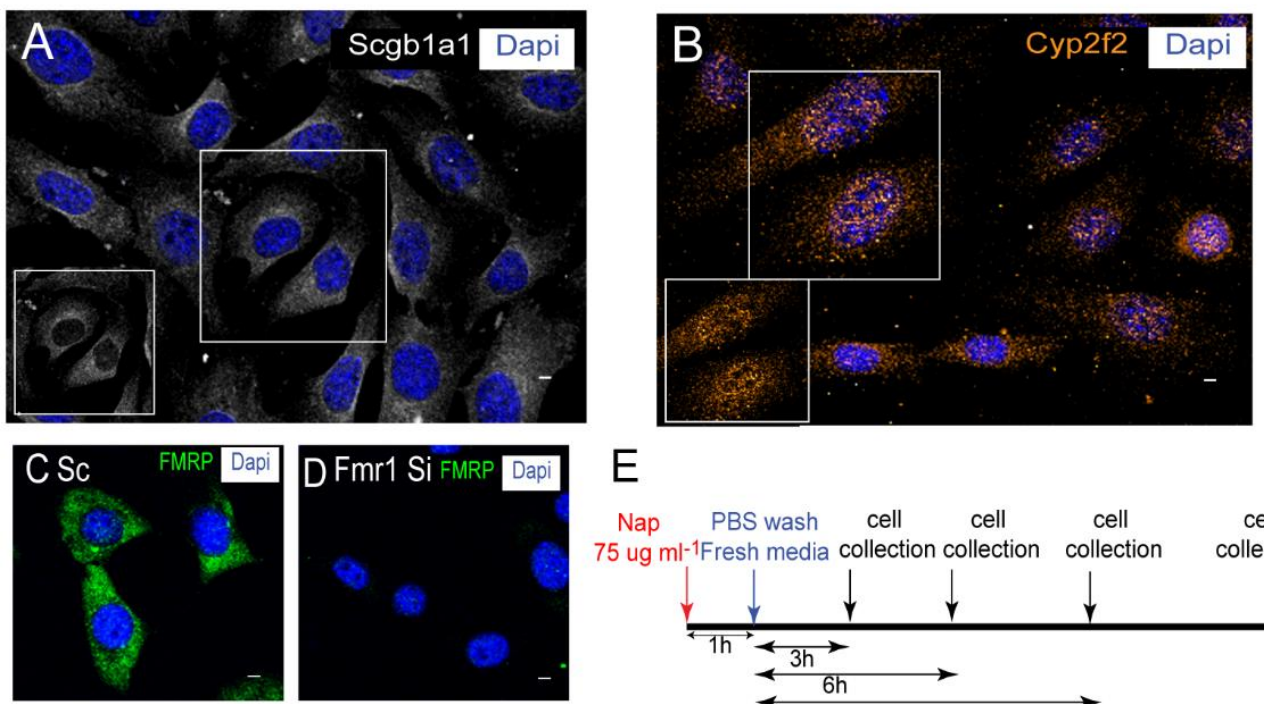

$E$

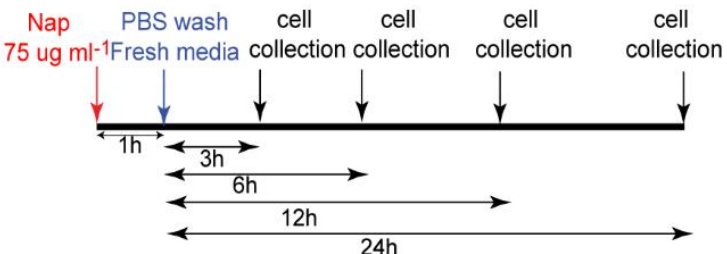

F

Un
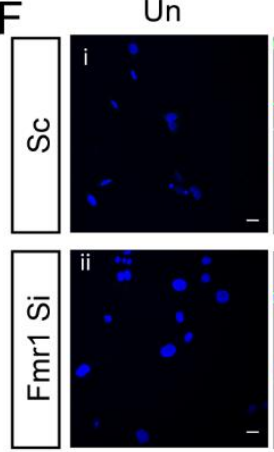

$\mathrm{H}$
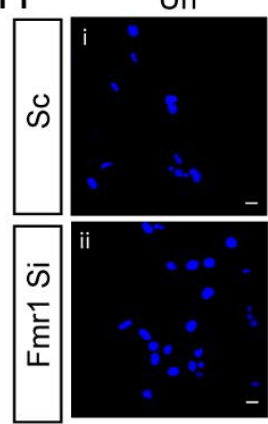

$6 \mathrm{~h}$
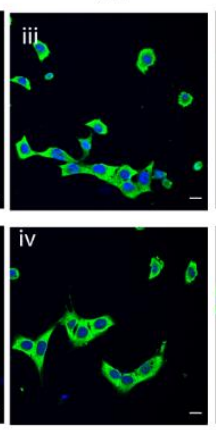

$6 h$
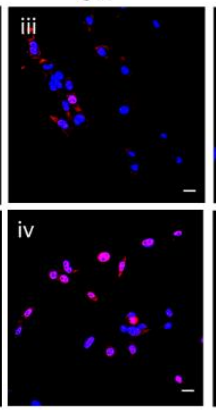

$24 \mathrm{~h}$
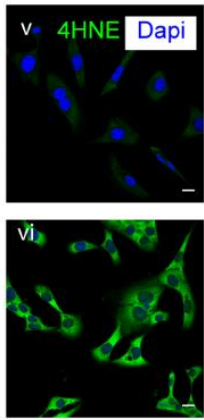

$24 \mathrm{~h}$
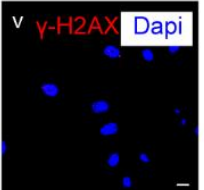

vî̀

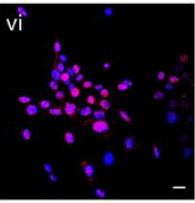

$\mathrm{G}$

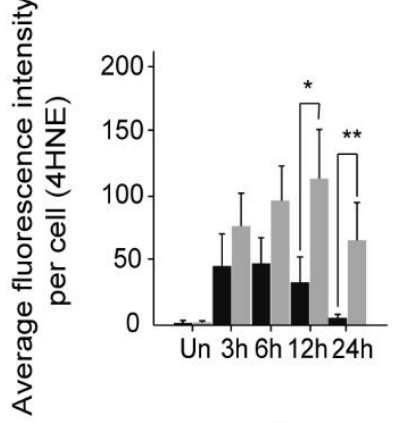

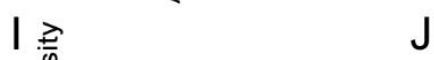

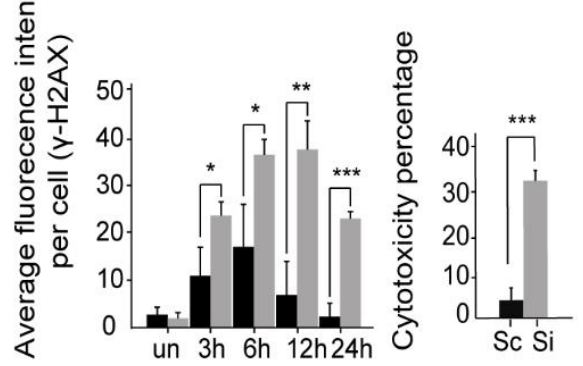


Figure-3

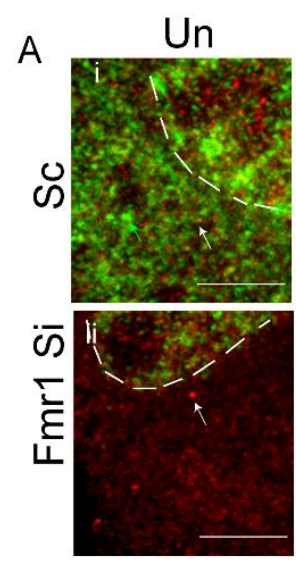

C

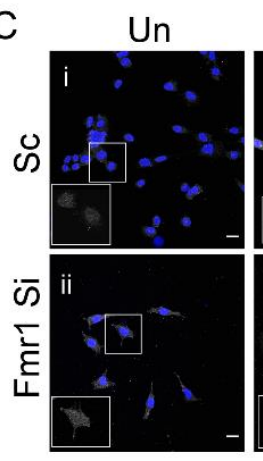

F

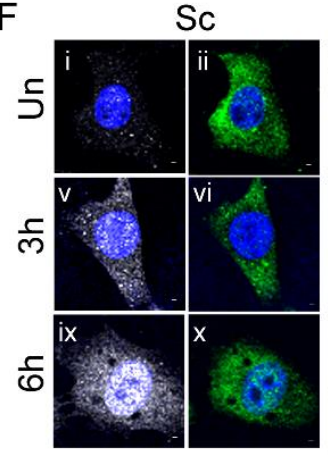

$3 \mathrm{~h}$
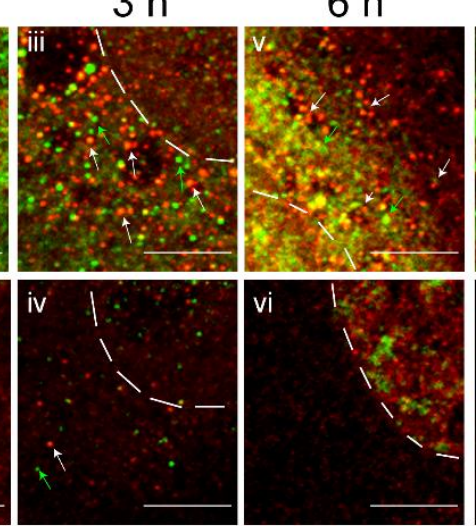

$3 \mathrm{~h}$

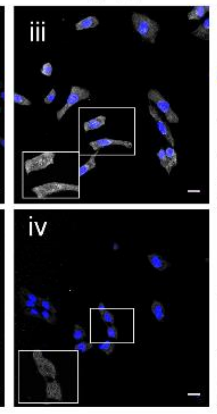

Atf4 Si

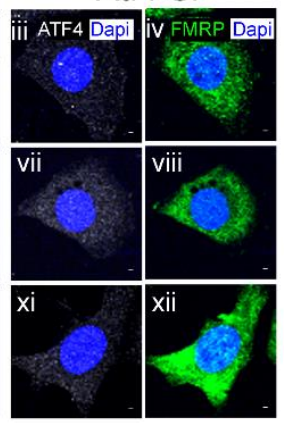

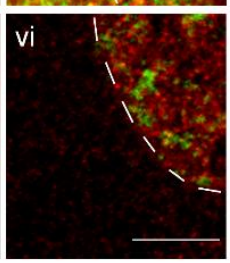

$6 \mathrm{~h}$

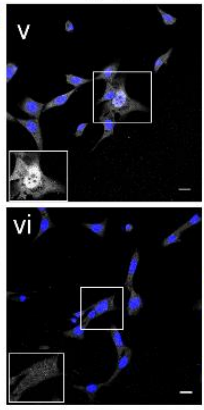

G

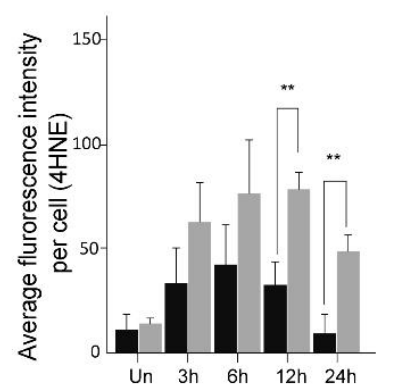

viii

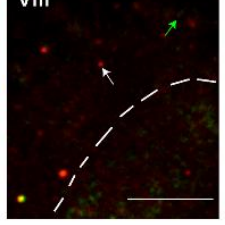

D

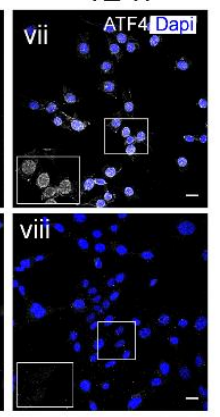

$24 \mathrm{~h}$
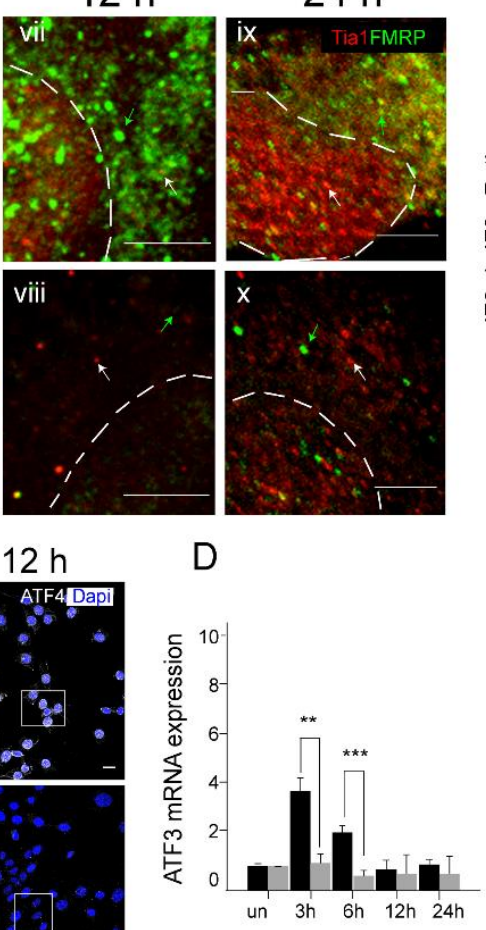

$\mathrm{H}$

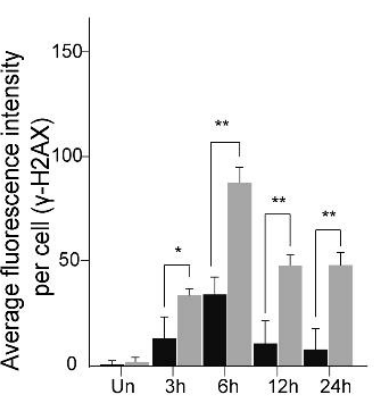

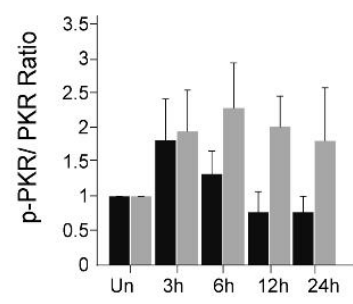

I

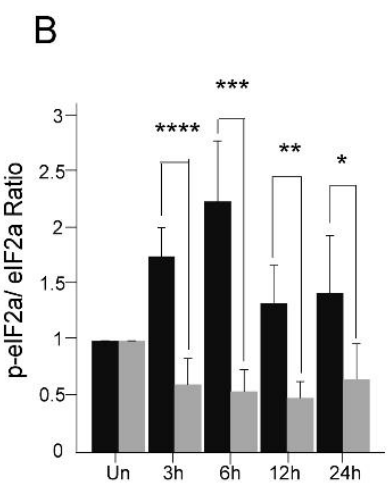

E

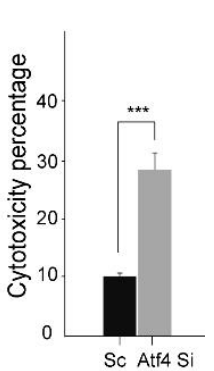


Figure-4
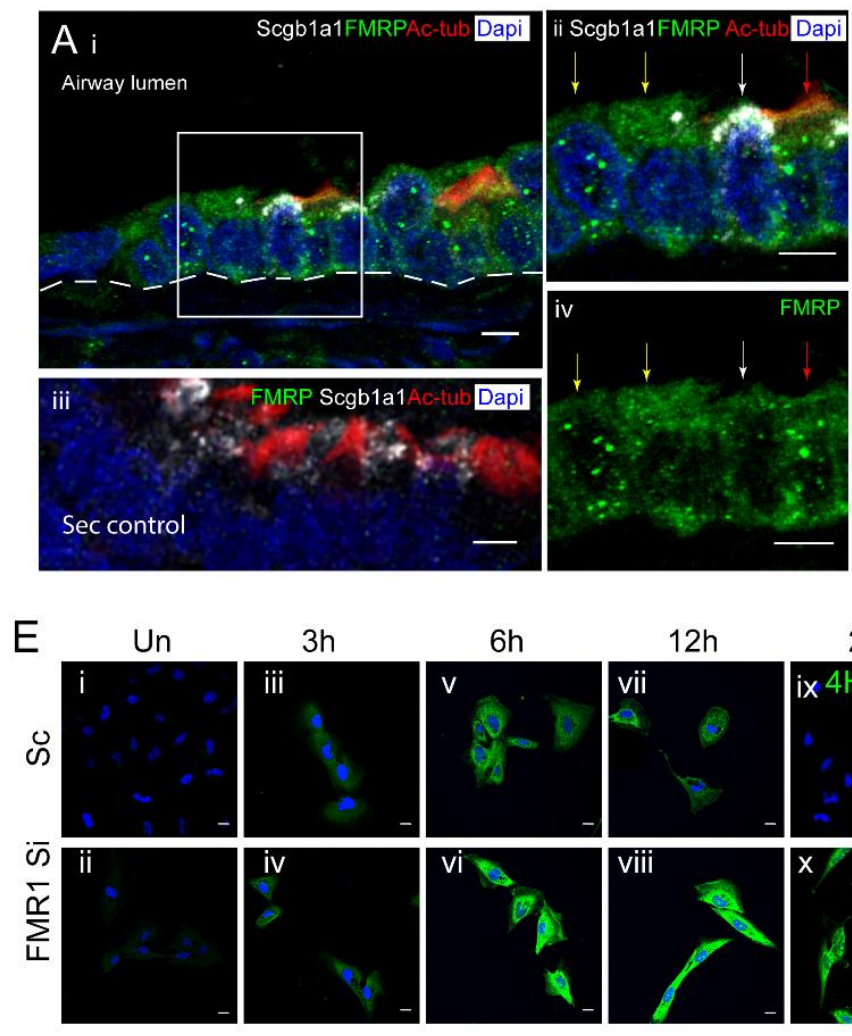

G

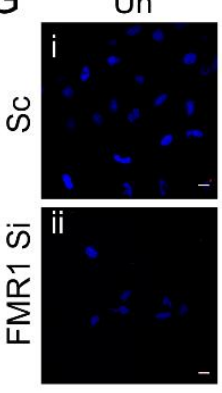

$3 \mathrm{~h}$

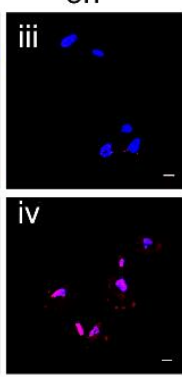

$6 \mathrm{~h}$

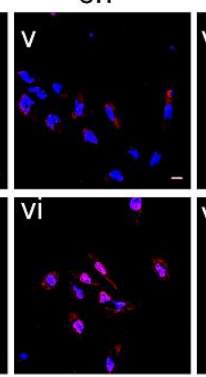

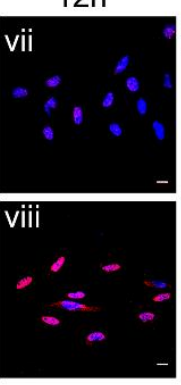

$24 \mathrm{~h}$
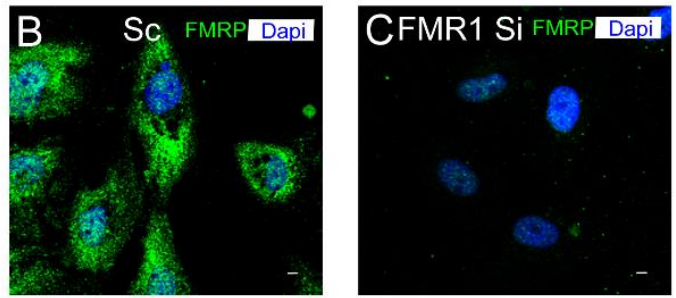

D
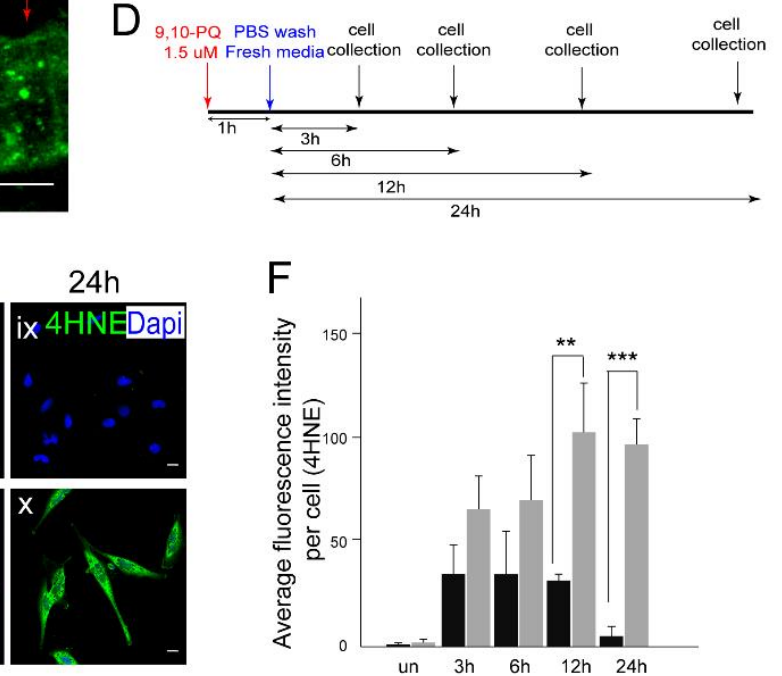

$24 \mathrm{~h}$
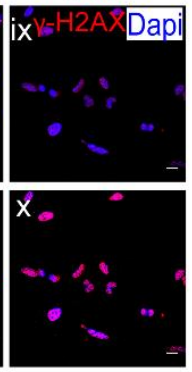

$\mathrm{H}$

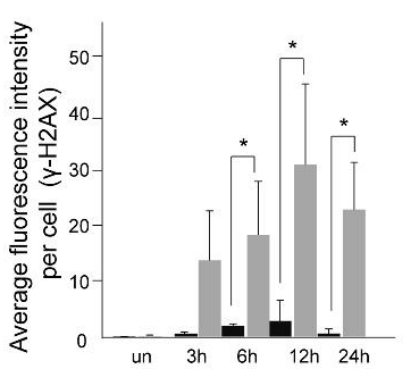

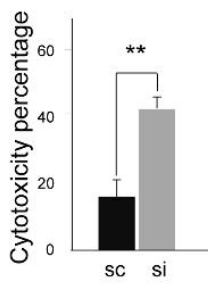


Figure-5

A
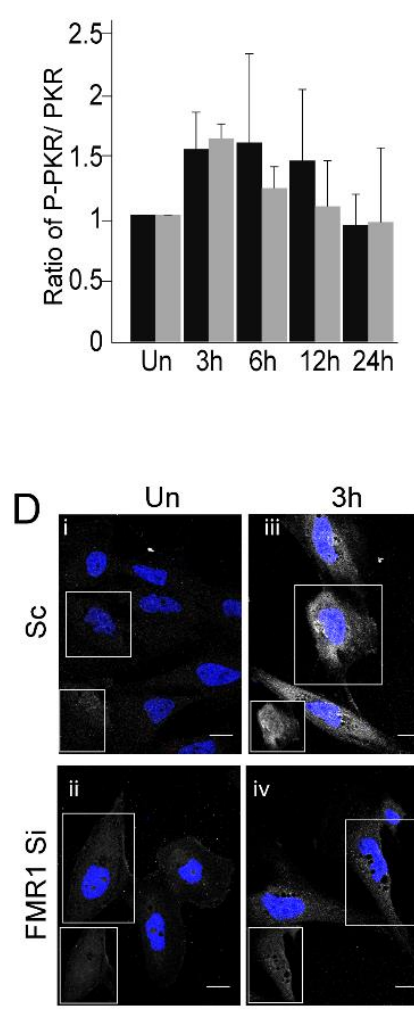

G

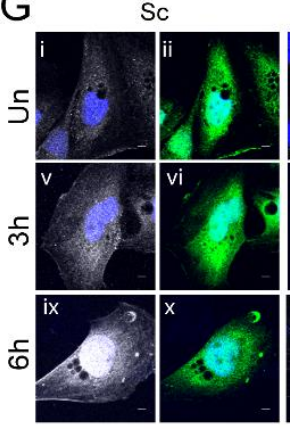

\section{B}

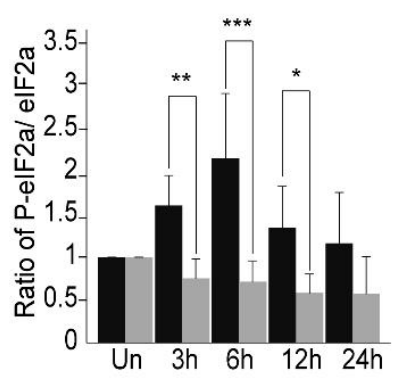

$6 h$
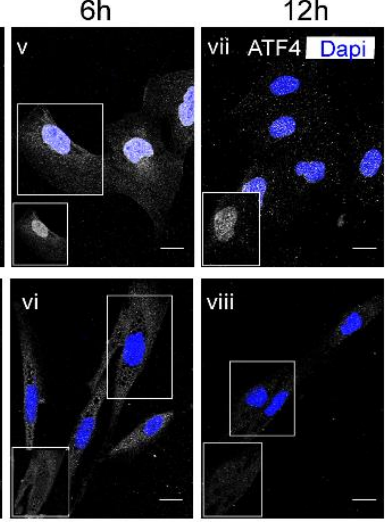

viii
C

¿
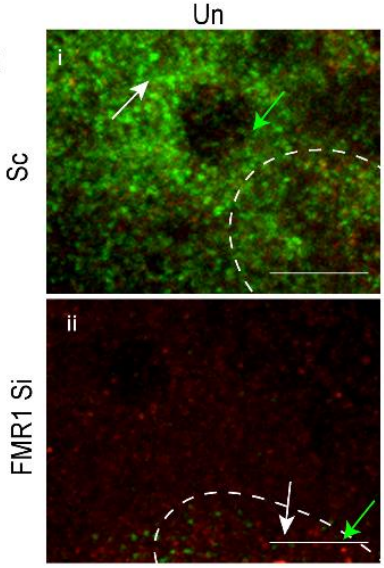

$E$
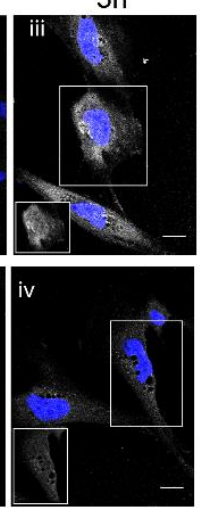

ATF4 Si

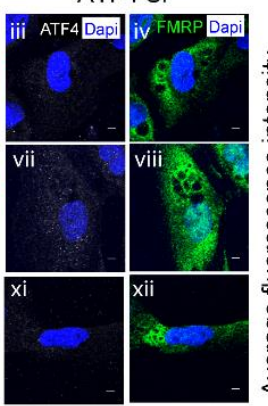

$\mathrm{H}$

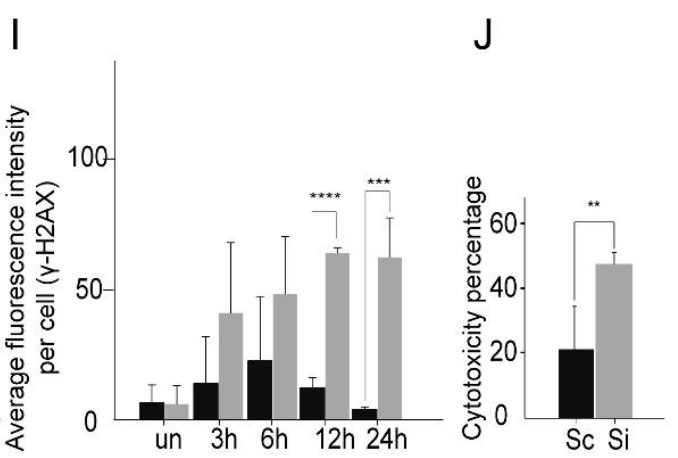


Figure-6

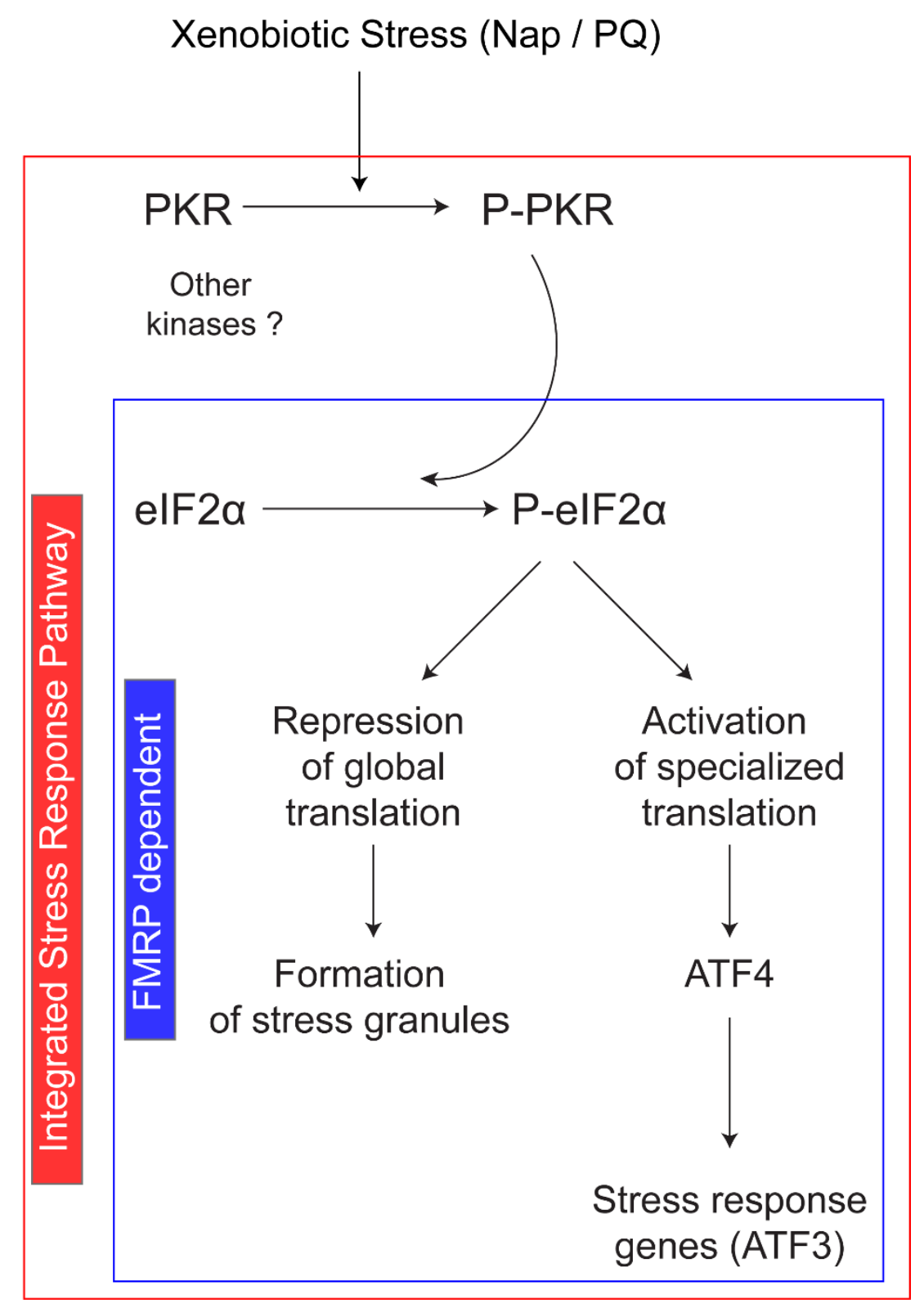


659 Figure 1: FMRP is expressed in the airways and more broadly and protects airway Club cells from Naphthalene induced stress. (A-D) FMRP expression in the murine lung. (A) Tiled image showing FMRP immunostaining (green, arrow) in the airway epithelium (demarcated by white dotted lines) and in the parenchyma of the murine lung. The airways are identified by expression of the Club cell (CC) marker Scgb1a1 (white, inset) and of the ciliated cell marker acetylated-Tubulin (red, inset). (B) Tiled image showing FMRP immunostaining in Fmr1 knockout (Fmr1 KO) mice. Note absence of FMRP (green) in both airway (demarcated by white dotted lines, inset) and parenchyma. (C-D) High resolution image of FMRP immunostaining (green) in airway epithelial cells. Shown here are CCs (white, white arrow in panel alongside) and ciliated cells (red, red arrow in panel alongside) in wild type (C) and Fmr1 KO (D). (E-H) Susceptibility of CCs to Naphthalene (Nap) injury in control and Fmr1 KO. (E) Schematic showing regimen for Nap injury. (F) Frequencies of Scgb1a1+ cells in wild type (black bars) and Fmr1 KO (grey bars) from uninjured (Un) and Nap-injured mice at different timepoints post injury. Data represents average + SD. (G-H) Expression of markers of oxidative (4HNE) and genotoxic ( $\mathrm{Y}-\mathrm{H} 2 \mathrm{AX})$ stress in airways from wild-type and Fmr1 KO mice prior to and post

675 Nap. (G i-G vi) 4HNE immunostaining (green) in the airways of wild-type (upper panel) and Fmr1 KO (lower panel) mice prior to and post Nap. (H i- H vi) Y-H2AX immunostaining 677 (red) in the airways of control (upper panel) and Fmr1 KO (lower panel) prior to and post 678 Nap. Also see Fig S1. Statistical significance was assessed by an unpaired two-tailed ttest (see methods, $\mathrm{p}<.05^{\star}, \mathrm{p}<.01^{* *}, \mathrm{p}<.001^{\star * *}$ ). The changes in the two groups over

680 time, across genotype and interaction parameters were also assessed by two-way ANOVA and found to be statistically significant. For Shapiro-Wilk normality test and twoway ANOVA see Table S1. Scale Bar=20 um.

Figure 2: FMRP deficient Club cell-like C22 cells are susceptible to Nap-induced stress. (A-D) Phenotypic characterization of C22 cells. (A) Scgb1a1 immunostaining (white) in C22 cells. (B) Cyp2f2 (orange) immunostaining in C22 cells. (C-D) FMRP immunostaining in $\mathrm{C} 22$ cells (C) and in C22 cells treated with Fmr1 siRNA (D). (E-J) 
687 Susceptibility of C22 cells to Nap (control (scrambled siRNA-treated, Sc) and Fmr1 688 siRNA-treated (Si)). (E) Schematic showing regimen for Nap injury. (F-I) Expression of markers of oxidative (4HNE) and genotoxic ( $\mathrm{Y}-\mathrm{H} 2 \mathrm{AX}$ ) stress in Sc and Si cells prior to and post Nap. (F i- F vi) 4HNE immunostaining (green) in Sc and Si cells prior to and post Nap. (G) Quantitation of 4HNE immunofluorescence per cell in Sc and Si cells prior to and post Nap ( $\geq 25$ cells were analysed per timepoint/per experiment, $n=3$ experiments). (H i- H vi) $\mathrm{y}-\mathrm{H} 2 \mathrm{AX}$ immunostaining (red) in Sc and Si cells prior to and post Nap. (I) Quantitation of $\mathrm{y}-\mathrm{H} 2 \mathrm{AX}$ immunofluorescence per cell in Sc and Si cells post Nap. (J) Cytotoxicity of Nap in Sc and Si cells 24 h post Nap ( $n=3$ experiments). Black bars (Sc), Grey bars (Si). Unpaired two-tailed t-test $\left(\mathrm{p}<.05^{\star}, \mathrm{p}<.01^{* *}, \mathrm{p}<.001^{\star \star *}\right)$. For normality test and two-way ANOVA see Table S2. Scale Bar=5 um.

698

699

700

701

702

703

704

705

706

707

708

709

710

711

712

713

714

715

Figure 3: FMRP deficient C22 cells fail to upregulate the Integrated Stress Response and induce ATF4, essential for protection from Nap-induced stress. (A i - A x) Expression of the stress granule marker Tia-1(white, arrows) in control (Scrambled siRNA-treated, Sc) and Fmr1 siRNA-treated (Si) cells prior to and post Nap. Note that FMRP expression in the same cells (green, arrow) does not completely overlap with Tia1. Also see Supplementary Figure 2 for Atx2/FMRP staining. (B) Western blot-based quantitation of phospho-elF2 $\alpha$ /elF2 $\alpha$ ratios in Sc and Si cells prior to and post Nap treatment ( $n=5$ experiments). See Fig. S2 for representative blots used for quantitation. (C i - C viii) ATF4 immunostaining in Sc (upper panel) and Si cells (lower panel) prior to and post Nap. Note nuclear accumulation of ATF4 in Sc cells by 6 h post Nap (inset). See Fig. S2 for quantitation. (D) Quantitative RT-PCR-based analysis of expression of the ATF4 target gene ATF3 in Sc and Si cells prior to and post Nap ( $n=3$ experiments). (E) Western blot-based quantitation of phospho-PKR/ PKR ratios in Sc and Si cells prior to and post Nap treatment ( $n=3$ experiments). See Fig S2 for representative blots used for quantitation. (F-I) Susceptibility of C22 cells to Nap in control (Scrambled siRNA-treated, Sc) and Atf4 siRNA-treated (Si) cells. (F i - F xii) Analysis of ATF4 levels (white) in Sc and Si cells prior to and post Nap treatment. Immunostaining for ATF4 (white) and FMRP (green) in Sc (left panel) and Si (right panel) cells. (G) Quantitation of 4HNE 
716 immunofluorescence per cell in Sc and Si cells prior to and post Nap. See Fig S2 for

717 representative images $(\mathbf{H})$ Quantitation of $\mathrm{y}-\mathrm{H} 2 \mathrm{AX}$ immunofluorescence per cell in Sc and 718 Si. See Fig. S2 for representative images. (I) Cytotoxicity of Nap in Sc and Si cells $24 \mathrm{~h}$ 719 post Nap exposure ( $\mathrm{n}=3$ experiments). Black bars (Sc), Grey bars (Si). Unpaired two720 tailed t-test $\left(p<.05^{\star}, p<.01^{* *}, p<.001^{* * *}\right)$. For normality test and two-way ANOVA see 721 Table S3. Scale Bar=5 um.

Figure 4: FMRP is expressed in the human airways and protects human bronchial BEAS-2B cells from 9, 10-Phenanthrenequinone-induced stress. (A-C) FMRP expression in the human lung and in BEAS-2B cells, a cell line derived from the human bronchial epithelium. (A i - A iv) FMRP immunostaining (green) in the distal airways of the human lung. (A $\mathbf{i}, \mathbf{A} \mathbf{i}, \mathbf{A}$ iv) Stained section showing FMRP expression in airway non-ciliated cells (Scgb1a1+ (white), white arrows; Scgb1a1-, yellow arrows) and ciliated cells (red, red arrow). Boxed area shown at higher magnification in top and bottom panels on the right. Negative control (secondary alone) for FMRP immunostaining shown in $\mathbf{A}$ iii. (B) FMRP immunostaining of BEAS-2B cells. (C) FMRP immunostaining of FMR1 siRNA-treated BEAS-2B cells. (D-I) Susceptibility of BEAS-2B cells to PQ injury in control (Scrambled siRNA-treated, Sc) and Fmr1 siRNA-treated (Si) cells. (D) Schematic showing regimen for $P Q$ injury. (E-H) Expression of markers of oxidative (4HNE) and genotoxic $(\mathrm{Y}-\mathrm{H} 2 \mathrm{AX})$ stress in Sc and $\mathrm{Si}$ cells prior to and post PQ. (E i - E x) 4HNE immunostaining (green) in Sc and Si cells prior to and post PQ. (F) Quantitation of 4HNE immunofluorescence per cell in Sc and Si cells post $P Q(\geq 25$ cells were analysed per timepoint/per experiment, $n=3$ experiments). ( $\mathbf{G} \mathbf{i}-\mathbf{G} \mathbf{x}$ ) $\mathbf{y}-\mathrm{H} 2 \mathrm{AX}$ immunostaining (red) in $\mathrm{Sc}$ and $\mathrm{Si}$ cells prior to and post PQ. (H) Quantitation of $\mathrm{y}-\mathrm{H} 2 \mathrm{AX}$ immunofluorescence per cell in Sc and Si cells post PQ. (I) Cytotoxicity of $P Q$ in Sc and Si cells $24 \mathrm{~h}$ post $P Q$ exposure ( $n=3$ experiments). Black bars (Sc), Grey bars (Si). Unpaired two-tailed t-test $\left(p<.05^{*}, p<.01^{* *}, p<.001^{* * *}\right)$. For normality test and two-way ANOVA see Table S4. Scale Bar=5 um. 
Figure 5: FMRP deficient BEAS-2B cells fail to upregulate the Integrated Stress Response and induce ATF4, essential for protection from 9, 10Phenanthrenequinone induced stress. (A) Western blot-based quantitation of phospho-PKR/ PKR ratios in Sc and Si cells prior to and post Nap treatment $(n=3$ experiments). See Fig. S4 for representative blots used for quantitation. (B) Western blotbased quantitation of phospho-elF2 $\alpha$ /elF2 $\alpha$ ratios in Sc and Si cells prior to and post PQ treatment ( $n=5$ experiments). See Fig. S4 for representative blots used for quantitation. (C i-C iv) Expression of the stress granule marker Tia-1(white, arrows) in control (Scrambled siRNA-treated, Sc) and Fmr1 siRNA-treated (Si) cells prior to and post PQ. Note that FMRP expression in the same cells (green, arrow) does not completely overlap with Tia-1. Also see Fig. S4 for G3BP/FMRP staining. (D-E) Analysis of ATF4 prior to and post PQ. (D) ATF4 immunostaining in Sc (upper panel) and Si cells (lower panel) prior to and post PQ treatment. Note nuclear accumulation of ATF4 in Sc cells by $6 \mathrm{~h}$ post $P Q$ treatment (inset). (E) Quantitation of ATF4 immunofluorescence per cell in Sc and $\mathrm{Si}$ cells prior to and post PQ. (F) Quantitative RT-PCR-based analysis of expression of ATF3 in Sc and Si cells prior to and post $P Q$ ( $n=3$ experiments). (G-J) Susceptibility of BEAS2B cells to $P Q$ in control (Scrambled siRNA-treated, Sc) and Atf4 siRNA-treated (Si) cells. (G i - G xii) Analysis of ATF4 levels (white) and FMRP (green) in Sc (left panel) and Si (right panel) cells prior to and post PQ treatment. Immunostaining for ATF4 (white) and FMRP (green) in Sc (left panel) and Si (right panel) cells. (H) Quantitation of 4HNE immunofluorescence per cell in Sc and Si. See Fig. S4 for representative images. (I) Quantitation of $\mathrm{y}-\mathrm{H} 2 \mathrm{AX}$ immunofluorescence per cell in Sc and Si. See Fig. S4 for representative images. $(\mathbf{J})$ Cytotoxicity of $P Q$ in $S c$ and Si cells $24 \mathrm{~h}$ post $P Q$ treatment $\left(n=3\right.$ experiments). Black bars (Sc), Grey bars (Si). Unpaired two-tailed t-test $\left(p<.05^{\star}, p<\right.$ $\left..01^{* *}, \mathrm{p}<.001^{* * *}\right)$. For normality test and two-way ANOVA see Table S5. Scale Bar=5 um.

\section{Figure 6: Model for the role of FMRP in the regulation of the Integrated Stress} Response in the lung. Exposure to xenobiotics such as Naphthalene (Nap) and 9,10Phenanthrenequinone (PQ) result in the activation of at least one of four stress- 
772 responsive kinases (PKR) and to the induction of Integrated Stress Response Pathway

773 (ISR, outlined in red). Our findings suggest that FMRP has an essential role downstream

774 to PKR phosphorylation (outline in blue). 